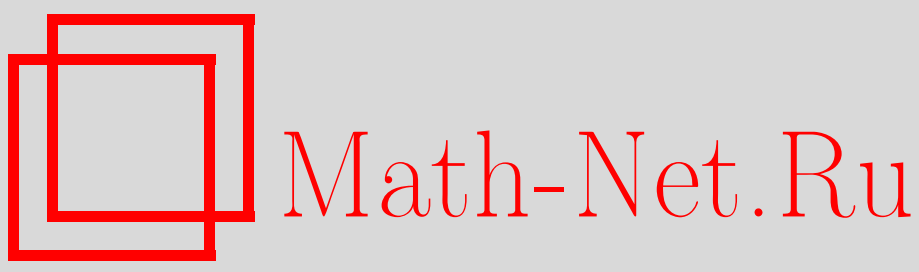

М. Я. Мазалов, О равномерных приближениях бианалитическими функциями на произвольных компактах в $\mathbb{C}$, Матем. сб., 2004, том 195, номер 5, 79-102

DOI: https://doi.org/10.4213/sm822

Использование Общероссийского математического портала Math-Net.Ru подразумевает, что вы прочитали и согласны с пользовательским соглашением http://www . mathnet.ru/rus/agreement

Параметры загрузки:

IP: 44.207 .124 .84

26 апреля 2023 г., 16:29:09 
УДК $517.538 .5+517.956 .2$

М.Я. Мазалов

\section{О равномерных приближениях бианалитическими функциями на произвольных компактах в $\mathbb{C}$}

Доказано, что каждая функция, непрерывная на произвольном компакте $X \subset \mathbb{C}$ и бианалитическая внутри $X$, может быть равномерно приближена на $X$ функциями, бианалитическими в окрестностях $X$.

Библиографоия: 17 названий.

\section{§ 0. Введение}

Пусть $\bar{\partial}$ - оператор Коши-Римана: $\bar{\partial}=\frac{1}{2}\left(\frac{\partial}{\partial x}+i \frac{\partial}{\partial y}\right)$. Бианалитическими функциями будем называть (следуя, например, [1]) решения уравнения $\bar{\partial}^{2} f(z)=0$ на открытых подмножествах комплексной плоскости $\mathbb{C}$ переменного $z=x+i y$. Напомним, что фундаментальньм решением оператора $\bar{\partial}^{2}$ является функция $\pi^{-1} \bar{z} / z$.

Цель работы - доказательство следующей теоремы 1 , которую в качестве гипотезы формулировал, в частности, Вердера [2], [3].

ТеОРема 1. Пусть $X-$ произвольный компакт в $\mathbb{C}, f(z)-$ произвольная функиия, непрерывная на $X$ и бианалитическая на множестве всех внутренних точек $X$. Тогда $f(z)$ равномерно приближсается на $X$ функииями, бианалитическими в окрестностях $X$.

Несколько точнее, приближающие функции строятся в виде следующих конечных линейных комбинаций:

$$
F(z)=\sum_{\iota} \lambda_{\iota} \frac{\overline{z-z_{\iota}}}{z-z_{\iota}}
$$

где $z_{\iota}$ - точки в дополнении $X$, а $\lambda_{\iota}$ - комплексные константы.

История вопроса рассматривается в [2] и [3]. Здесь кратко отметим следующее $\left[2 ;\right.$ п. 1]. В силу ограниченности фундаментального решения оператора $\bar{\partial}^{2}$ теорема 1 является естественным аналогом теорем Витушкина [4] и Келдыша-Дени [5], [6] о равномерном приближении соответственно в классах аналитических и гармонических функций. Ранее был установлен ряд частных случаев теоремы 1: Трент и Ванг [7] - компакт $X$ нигде не плотен; Кармона [8] - внутренняя граница $X$ пуста; Ванг [9] - внутренняя граница $X$ не более чем счетна; Вердера [2] модуль непрерывности функции $f(z)$ удовлетворяет условию Дини; Мазалов [10]в каждой внутренней точке $X$ выполнено условие $\bar{\partial} f(z)=0$.

Автор глубоко признателен М. С. Мельникову и П. В. Парамонову за внимание к работе и ряд полезных замечаний.

(C) М.Я. МАзАлов 2004 


\section{§1. Обозначения. Подготовительные результаты}

На протяжении всей статьи буквами $A, A_{0}, A_{1}, A_{2}, \ldots$ будем обозначать положительные абсолютные константы. Каждая из этих констант в разных соотношениях может принимать различные значения, причем существенны не сами конкретные значения, а только факт их существования.

Пусть $B=B\left(z_{0}, r\right)$ - замкнутый круг с центром $z_{0}$ и радиусом $r$, при этом для произвольного $\alpha>0$ через $\alpha B$ обозначим круг с тем же центром и радиусом $\alpha r$. Говоря о квадратах, всегда будем иметь в виду квадраты со сторонами, параллельными осям координат. Для квадрата $Q$ через $d(Q)$ обозначим длину его стороны; квадрат с тем же центром, что и $Q$, и стороной $\alpha d(Q)$ обозначим $\alpha Q$. Замкнутыми двоичными квадратами будем называть квадраты $Q_{k}^{n, l}$ следуюшего вида: $Q_{k}^{n, l}=\left[n 2^{-k},(n+1) 2^{-k}\right] \times\left[l 2^{-k},(l+1) 2^{-k}\right]$ при целых $k, n$ и $l$.

Для произвольного компакта $E$ множество его внутренних точек обозначим через $\stackrel{\circ}{E}$. Функцию $\varphi(z)$ с носителем $\operatorname{supp} \varphi(z)$, принадлежащим кругу $B=B\left(z_{0}, r\right)$, будем называть стандартной относительно $B$, если она неотрицательная, дважды гладкая, причем $\left|\nabla^{l} \varphi(z)\right| \leqslant A r^{-l}$ для всех $z$ и $l=0,1,2$. (Функция, стандартная относительно некоторого квадрата, определяется аналогично.)

Пусть $\Phi(z)$ - функция, бианалитическая всюду вне ограниченного множества. Если найдется $\lambda \in \mathbb{C}$, при котором $\lim _{z \rightarrow \infty}(\Phi(z)-\lambda \bar{z} / z)=0$, запишем это как $\Phi(z) \sim \bar{z} / z$.

Следующие интегральное представление (1.1) и оценки (леммы 1.1) являются аналогами фактов, хорошо известных для различных классов эллиптических уравнений $[4 ;$ гл. 1, 2], [11; п. 1В], [12; пп. 3, 4].

Пусть $\Phi(z)$ - функция, непрерывная в $\mathbb{C}$ и бианалитическая вне некоторого компакта $E$, причем $\Phi(z) \sim \bar{z} / z$. Тогда для любого $z \in \mathbb{C}$ справедливо представление, которое понимается в обобщенном смысле:

$$
\Phi(z)=\pi^{-1} \frac{\bar{z}}{z} *\left(\varphi_{E} \bar{\partial}^{2} \Phi\right)
$$

где $\varphi_{E}-$ произвольная дважды гладкая функция с компактным носителем такая, что $\varphi_{E} \equiv 1$ в некоторой окрестности $E ; *$ - свертка.

Напомним доказательство формулы (1.1). Пусть

$$
r(z)=\Phi(z)-\pi^{-1} \frac{\bar{z}}{z} *\left(\varphi_{E} \bar{\partial}^{2} \Phi\right)
$$

Тогда $\bar{\partial}^{2} r \equiv 0$ (в обобщенном смысле). В силу эллиптичности оператора $\bar{\partial}^{2}$ тождество $\bar{\partial}^{2} r \equiv 0$ справедливо всюду в $\mathbb{C}$. При этом выполнено условие $r(z) \sim \bar{z} / z$ и, следовательно, функция $r(z)$ равномерно ограничена в $\mathbb{C}$. В силу аналога теоремы Лиувилля (для бианалитических функций, например, [1; теорема 2.2]) получим, что $r(z) \equiv 0$.

Лемма 1.1. Пусть $E$ - компакт, $E \subset B=B\left(z_{0}, r\right) ; M>0 ; \Phi(z)-\oint у н к-$ иия, непрерывная в $\mathbb{C}$, бианалитическая вне $E$ и такая, что $\Phi(z) \sim \bar{z} / z u$ $|\Phi(z)| \leqslant M$ в круге 2 B. Тогда (например, $[2 ;$ п. 5]): 
1) вне круга В функция $\Phi(z)$ представима обобщенныцм рядом Лорана (сходящимся в $\left.C^{\infty}(\mathbb{C} \backslash B)\right)$ :

$\Phi(z)=c_{0} \frac{\overline{z-z_{0}}}{z-z_{0}}+\frac{b_{1}}{z-z_{0}}+c_{1} \frac{\overline{z-z_{0}}}{\left(z-z_{0}\right)^{2}}+\cdots+\frac{b_{n}}{\left(z-z_{0}\right)^{n}}+c_{n} \frac{\overline{z-z_{0}}}{\left(z-z_{0}\right)^{n+1}}+\cdots ;$

2) лорановские коэффициенты вычисляются по формулам:

$$
c_{n}=\pi^{-1}\left\langle\bar{\partial}^{2} \Phi,\left(z-z_{0}\right)^{n} \varphi_{E}\right\rangle, \quad b_{n}=-\pi^{-1}\left\langle\bar{\partial}^{2} \Phi, \overline{z-z_{0}}\left(z-z_{0}\right)^{n-1} \varphi_{E}\right\rangle
$$

где $n=0,1, \ldots, \varphi_{E}-$ функиия, имеющая тот же смысл, что и в формуле (1.1), угловые скобки означают действие распределения порядка 2 с компактным носителем на дважды гладкую функцию;

3) справедливы оценки: $\max \left(\left|c_{n}\right|,\left|b_{n}\right|\right) \leqslant A M(2 r)^{n}, n=0,1, \ldots$;

4) пусть найдется число $k \in \mathbb{N}$ такое, что при всех $n<k \quad c_{n}=0$ и $b_{n}=0$, тогда всюду в $\mathbb{C}$ выполнена оценка:

$$
|\Phi(z)| \leqslant A M \min \left(1, \frac{(3 r)^{k}}{\left|z-z_{0}\right|^{k}}\right)
$$

причем коэффициенты $c_{k}$ и $b_{k}$ не зависят от $z_{0}$ (в частности, $|\Phi(z)| \leqslant A M$ и с никогда не зависит от $\left.z_{0}\right)$;

5) пусть $\varphi_{1}(z)$ - функция, стандартная относительно круга $B_{1}=B\left(z_{1}, \delta\right)$, а $\omega$ - колебание $\Phi(z)$ в круге $B_{1}$, тогда функция

$$
\Phi_{\delta}(z)=\pi^{-1} \frac{\bar{z}}{z} *\left(\varphi_{E} \varphi_{1} \bar{\partial}^{2} \Phi\right)
$$

непрерывна в $\mathbb{C}$, бианалитична вне мнохсества $E \cap B_{1}$ (если $E \cap B_{1}=\varnothing$, то $\left.\Phi_{\delta}(z) \equiv 0\right) u\left|\Phi_{\delta}(z)\right| \leqslant A \omega$ в $\mathbb{C}$.

Заметим, что оценки 3 ) леммы 1.1 сразу же следуют из формул 2), если взять функцию $\varphi_{E}(z)$ стандартной относительно круга $\left.2 B ; 4\right)$ следует из 3$)$ так же, как и в доказательстве леммы $1[4 ;$ гл. 2, п. 4$] ; 5)$ доказывается так же, как и в лемме 1 [4; гл. 2, п. 3].

В следуюшем вспомогательном утверждении рассматриваются некоторые свойства приближающих функций (0.1).

ЛЕмма 1.2. Пусть функиия $U_{1}(z)$ бианалитична в некоторой окрестности круга $B\left(z_{0}, r\right), M>0, \lambda \in \mathbb{C}$ и при этом для функиии $U(z)$ :

$$
U(z)=U_{1}(z)+\lambda \frac{\overline{z-z_{0}}}{z-z_{0}}
$$

выполнена оценка $|U(z)| \leqslant M$ в кольце $K=B \backslash 0.5 B$.

Тогда верны оченки $|\lambda| \leqslant A M u|U(z)| \leqslant A M$ в круге $B$. 
ДокАЗАТЕЛЬСТво. Пусть $\varphi(z)$ - функция, стандартная относительно $B$, причем $\varphi \equiv 1$ на $0.5 B$. Ясно, что $\lambda=\pi^{-1}\left\langle\bar{\partial}^{2}\left(U-U_{1}\right), \varphi\right\rangle=\pi^{-1}\left\langle\bar{\partial}^{2} U, \varphi\right\rangle$ или

$$
\lambda=\pi^{-1} \iint_{K} U(z) \bar{\partial}^{2} \varphi(z) d x d y .
$$

Отсюда следует, что $|\lambda| \leqslant A M$ и $\left|U_{1}(z)\right| \leqslant A_{1} M$ в кольце $K$. Из стандартных оценок решений эллиптических уравнений внутри области (например, [1; теорема 1.5]) получим: $\left|U_{1}(z)\right| \leqslant A_{2} M$ в $B$. Следовательно, $|U(z)| \leqslant A M$ в $B$. Лемма доказана.

При доказательстве теоремы 1 сушественно используются схема А. Г. Витушкина разделения особенностей и приближения функции по частям [4; гл. 1,2$]$ и метод группировки индексов П.В. Парамонова [12].

Сначала, используя теорему Уитни [13; гл. 6, п. 2.2], продолжим исходную функцию $f(z)$ за границу $X$ до функции, непрерывной в $\mathbb{C}$ и с компактньм носителем.

Пусть $\omega_{f}(\delta)-$ модуль непрерьвнности продолженной функции $f(z)$. Зафиксируем $\delta>0$. Проведем разбиение единицы в $\mathbb{C}$ с помощью функций $\varphi_{j}(z)$, стандартных относительно соответствуюших кругов $B_{j}$. Здесь $j=\left(j_{1}, j_{2}\right), j_{1}$ и $j_{2}$ целые, $B_{j}=B\left(j_{1} \delta+i j_{2} \delta, \delta\right)$ (по поводу построения таких разбиений единицы см. [14; c. 102], а также [4; гл. 2 , п. 1]). В силу (1.1) получим:

$$
f(z)=\pi^{-1} \frac{\bar{z}}{z} *\left(\bar{\partial}^{2} f\right)=\sum_{j} f_{j}(z)
$$

где

$$
f_{j}(z)=\pi^{-1} \frac{\bar{z}}{z} *\left(\varphi_{j} \bar{\partial}^{2} f\right)
$$

Пусть $\stackrel{\circ}{X}$-множество внутренних точек исходного компакта $X, E_{j}$-следующие компакты:

$$
E_{j}=B_{j} \backslash \stackrel{\circ}{X}
$$

Как вытекает из леммы $1.1,5)$, каждая функция $f_{j}(z)$ бианалитична вне соответствуюшего компакта $E_{j}$, непрерывна в $\mathbb{C}$ и $\left|f_{j}(z)\right| \leqslant A \omega_{f}(\delta)$. Если $B_{j} \subset \stackrel{\circ}{X}$, то $f_{j}(z) \equiv 0$, причем $f_{j}(z) \not \equiv 0$ лишш для конечного числа индексов $j$.

ЛЕмма 1.3. Для каждой функции $f_{j}(z)$ из (1.4) существует функиия $F_{j}(z)$ вида (0.1) такая, что:

1) все точки $z_{\iota}$ принадлежат открытому множеству $2 \stackrel{\circ}{B_{j}} \backslash X$;

2) $\left|F_{j}(z)\right| \leqslant A_{1} \omega_{f}(\delta)$;

3) $\left|\lambda_{\iota}\right| \leqslant A_{2} \omega_{f}(\delta)$ для всех номеров $\iota$;

4) имеет место асимптотика:

$$
f_{j}(z)-F_{j}(z)=O\left(|z|^{-2}\right) .
$$

Пояснения. Указанная асимптотика означает, что у функций $f_{j}$ и $F_{j}$ совпадают лорановские коэффициенты $c_{0}, b_{1}$ и $c_{1}$ из (1.2) (при разложении с обшим центром). Как следует из леммы 1.2, условие 3 ) леммы 1.3 является следствием условия 2). 
Заметим, что стандартный вариант схемы Витушкина [4] требовал бы в лемме 1.3 вместо (1.6) выполнения для каждого индекса $j$ следующего условия:

$$
f_{j}(z)-F_{j}(z)=O\left(|z|^{-3}\right) .
$$

Тогда из леммы $1.1,4)$, примененной при $k=3$ к разностям $f_{j}-F_{j}$ так же, как и в лемме $1\left[4 ;\right.$ гл. 2, п. 4], сразу следовало бы $\left|f(z)-\sum_{j} F_{j}(z)\right| \leqslant A \omega_{f}(\delta)$. Однако в условии (1.7) необходимости нет. Теорема 1 выводится из леммы 1.3 с помощью группировки индексов.

Метод группировки индексов предложен Парамоновым в [12] (см. также [14]). В $[10 ;$ п. 5] указанный метод был адаптирован к случаю равномерного приближения в классе бианалитических функций. Суть метода (в данной ситуации): используя условие (1.6), добиться вьполнения условия, соответствуюшего (1.7), не для каждого индекса, а для специальным образом скомбинированных групп индексов (так называемых nолных груnn). Оказывается, что оставшихся (несгруппированных) индексов “достаточно мало” и для них можно обойтись оценкой (1.6). При этом оценок (аналогичных $[14 ;$ с. 109,110$])$ оказывается вполне достаточно для равномерного приближения $f(z)$.

Заметим, что хотя в $[10 ;$ п. 5$]$ метод применяется для доказательства частного случая теоремы 1 (в котором предполагается, что $f(z)$ аналитична в $\stackrel{\circ}{X}$ ), все рассуждения и оценки $[10 ;$ п. 5] переносятся на общий случай дословно, без каких-либо изменений.

Таким образом, для справедливости теоремы 1 достаточно доказать лемму 1.3, которую, в свою очередь, сведем к основной лемме 1.4.

Вернемся к формулировке леммы 1.3. Рассмотрим индекс $j$ такой, что $f_{j}(z) \not \equiv 0$. Так как функция $f_{j}(z)$ непрерывна, то компакт $E_{j}$ из (1.5) содержит бесконечное множество точек. Пусть $t_{1}$ и $t_{2}$ - наиболее удаленные друг от друга точки $E_{j}$, а $\delta_{1}=\left|t_{1}-t_{2}\right|$. Рассмотрим $z_{1}^{\prime}$ и $z_{2}^{\prime}$ - точки, принадлежашие множеству $2 \stackrel{\circ}{B}_{j} \backslash X$ и такие, что расстояния $\left|t_{1}-z_{1}^{\prime}\right|$ и $\left|t_{2}-z_{2}^{\prime}\right|$ "очень малы" по сравнению с $\delta_{1}$.

Используя точки $z_{1}^{\prime}$ и $z_{2}^{\prime}$, нетрудно построить функцию вида

$$
\lambda_{1} \overline{\frac{z-z_{1}^{\prime}}{z-z_{1}^{\prime}}}+\lambda_{2} \frac{\overline{z-z_{2}^{\prime}}}{z-z_{2}^{\prime}}
$$

удовлетворяюшую условиям 2$), 3$ ) леммы 1.3 и уравниваюшую у $f_{j}$ коэффициент $c_{0}$ и некоторую линейную комбинацию коэффициентов $c_{1}$ и $b_{1}$, зависяшую от направления прямой, проходящей через $z_{1}^{\prime}$ и $z_{2}^{\prime}$. Действительно, рассмотрим функции следуюшего вида:

$$
K\left(z, z_{1}, z_{2}\right)=\frac{\overline{z-z_{2}}}{z-z_{2}}-\frac{\overline{z-z_{1}}}{z-z_{1}} .
$$

Чтобы "расслоить" коэффициенты $b_{1}$ и $c_{1}$ по вешественным направлениям, введем обозначения:

$$
c_{x}=c_{1}+b_{1}, \quad c_{y}=i\left(b_{1}-c_{1}\right) .
$$


Ясно, что при этом:

$$
\frac{b_{1}}{z-z_{0}}+c_{1} \frac{\overline{z-z_{0}}}{\left(z-z_{0}\right)^{2}} \equiv \frac{c_{x}\left(x-x_{0}\right)+c_{y}\left(y-y_{0}\right)}{\left(z-z_{0}\right)^{2}} .
$$

Нетрудно заметить, что для вешественных $a$ и $b$ :

$$
\frac{\overline{z-a}}{z-a}-\frac{\bar{z}}{z}=-2 i a \frac{y}{z(z-a)}, \quad \frac{\overline{z-i b}}{z-i b}-\frac{\bar{z}}{z}=2 i b \frac{x}{z(z-i b)}
$$

Отсюда следует:

$$
c_{x}\left(K\left(z, z_{1}, z_{2}\right)\right)=2 i \operatorname{Im}\left(z_{2}-z_{1}\right), \quad c_{y}\left(K\left(z, z_{1}, z_{2}\right)\right)=-2 i \operatorname{Re}\left(z_{2}-z_{1}\right) .
$$

Повернем систему координат на плоскости так, чтобы прямая $\left(z_{1}^{\prime}, z_{2}^{\prime}\right)$ оказалась параллельной оси $O x$. Тогда при разложении в ряд Лорана с центром $z_{1}^{\prime}$ для функции $K_{0}(z)=\frac{\overline{z-z_{1}^{\prime}}}{z-z_{1}^{\prime}}$ будут выполнены условия: $c_{0}=1$ и $c_{x}=c_{y}=0$, а для $K\left(z, z_{1}^{\prime}, z_{2}^{\prime}\right)$ в силу (1.10) соответственно $c_{0}=c_{x}=0$ и $\left|c_{y}\right| \geqslant A \delta_{1}$. Тем самым (в силу леммы 1.1), линейная комбинация $K_{0}(z)$ и $K\left(z, z_{1}^{\prime}, z_{2}^{\prime}\right)$ позволяет с соответствующими оценками уравнять у исходной функции $f_{j}$ коэффициенты $c_{0}$ и $c_{y}$, не изменяя коэффициента $c_{x}$. Поэтому задача сводится $к$ построению функции, удовлетворяющей условиям 1)-3) леммы 1.3 и уравнивающей у $f_{j}$ всего лишь один коэффициент $c_{x}$.

Сформулируем соответствуюшее утверждение в более удобной форме, чтобы избежать несушественных деталей (это и будет лемма 1.4). Ряд пояснений к формулировке леммы 1.4 сделаем ниже.

ЛЕмма 1.4 (основная). Пусть $Q_{0}-$ единичный квадрат: $Q_{0}=[0,1] \times[0,1]$, $D_{0}=3 Q_{0}$. Пусть $\stackrel{\circ}{E}$ - непустое открытое подмножество квадрата $Q_{0}$, $E$ - замыкание $\stackrel{\circ}{E}, \Phi(z)-$ функиия, непрерывная в $\mathbb{C}$, бианалитическая всюду вне $E$, причем $|\Phi(z)| \leqslant 1$ на $D_{0} u \lim _{z \rightarrow \infty} \Phi(z)=0$.

Тогда существуют конечный набор точек $z_{\iota} \in \stackrel{\circ}{E}$ и абсолютная константа $A_{0}>0$ такие, что в классе функиий $G(z)$, удовлетворяющих условиям 1) и 2):

1) $G(z)=\sum_{\iota} \lambda_{\iota} \frac{\overline{z-z_{\iota}}}{z-z_{\iota}}$, де $\lambda_{\iota}-$ комплекснье константьи, причем $\sum_{\iota} \lambda_{\iota}=0$;

2) $|G(z)| \leqslant 1$ на $D_{0}$,

найдется такая функция, для которой выполнено условие:

$$
\left|c_{x}(G)\right| \geqslant A_{0}\left|c_{x}(\Phi)\right|
$$

Пояснения. $\mathrm{C}$ помощью преобразования подобия компакт $E_{j}$ формулы (1.5) “пересажен” в квадрат $Q_{0}$. При этом открытое подмножество $\stackrel{\circ}{E}$ квадрата $Q_{0}$ из леммы 1.4 имеет смысл $2 \stackrel{\circ}{B}{ }_{j} \backslash X$ и, следовательно, $E_{j}$ является замыканием некоторого открытого подмножества $\stackrel{\circ}{E}$, в частности подмножеством компакта $E$ из леммы 1.4. Функция $\Phi(z)$ (с точностью до умножения на константу) имеет 
смысл $f_{j}(z)-c_{0}\left(f_{j}\right) \widehat{K_{0}(z)}$, где $\widehat{K_{0}(z)}-$ непрерывная функция, совпадающая с $K_{0}(z)$ вне "очень маленького" круга $B\left(z_{1}^{\prime}, r\right)$, принадлежашего $2{ }^{\circ}{ }_{j} \backslash X$, а при $z \in B\left(z_{1}^{\prime}, r\right)$ равная, например, $\left.\left(\overline{\left(z-z_{1}^{\prime}\right.}\right) / r\right)^{2}$. Таким образом, $E$ "не меньше", чем множество особенностей функции $f_{j}(z)-c_{0}\left(f_{j}\right) \widetilde{K_{0}(z)}$. Условие $\sum_{\iota} \lambda_{\iota}=0$, очевидно, равносильно следуюшему: $\lim _{z \rightarrow \infty} G(z)=0$. Так как $\lim _{z \rightarrow \infty} \Phi(z)=0$ и $\lim _{z \rightarrow \infty} G(z)=0$, то (лемма $\left.\left.1.1,4\right)\right)$ коэффициенты $c_{x}$ указанных функций не зависят от центра разложения в ряд Лорана. Функция, уравнивающая у $\Phi(z)$ коэффициент $c_{x}$, имеет вид $\lambda G(z)$, где $|\lambda| \leqslant A$. Набор точек $z_{\iota}$ зависит от геометрического строения компакта $E$ и модуля непрерьвности функции $\Phi(z)$.

Напомним также, что в силу лемм 1.1 и 1.2 из $|G(z)| \leqslant 1$ на $D_{0}$ следует, что $|G(z)| \leqslant A$ в $\mathbb{C}_{\text {и }}\left|\lambda_{\iota}\right| \leqslant A$ для всех номеров $\iota$.

Лемма 1.4 доказывается в следующих параграфах. В 22 дается общая схема доказательства, а в $\S \S 3$ и 4 доказываются все вспомогательные утверждения.

Следуюшая техническая лемма установлена в [10; п. 4]. Она используется в дальнейшем (лемма 2.2) для геометрической оценки снизу $\sup \left|c_{x}(G)\right|$ по классу функций $G$ леммы 1.4. Эта оценка основана на формуле (1.10).

Лемма 1.5 [10; лемма 3.1]. Пусть для любого натурального $n \Gamma^{\prime}$ - ломаная с вериинами $t_{s}=\tau_{s}+i \nu_{s}, s=1, \ldots, n, \tau_{1}<\cdots<\tau_{n}$, являющаяся графиком некоторй функиии $y=Y(x)$ с $\left|Y^{\prime}(x)\right| \leqslant 1 / 4$.

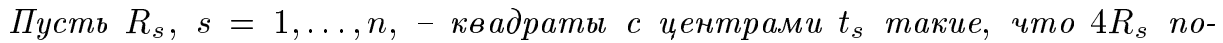
парно не пересекаются. Пусть для соответствующего $s\left[z_{s}, z_{s}^{\prime}\right]$ - отрезок с иентром $t_{s}$, принадлежсащий $R_{s}$, такой, что $\left|\operatorname{Im}\left(z_{s}-z_{s}^{\prime}\right)\right|=\frac{1}{5} d\left(R_{s}\right)$.

Тогда существуют константы $\lambda_{s}, s=1, \ldots, n,\left|\lambda_{s}\right| \leqslant 1$, функиия $G_{0}(z)$ и абсолютная константа $A>0$ такие, что:

1) $G_{0}(z)=\sum_{s=1}^{n} \lambda_{s} K\left(z, z_{s}, z_{s}^{\prime}\right)\left(\right.$ әде $K\left(z, z_{s}, z_{s}^{\prime}\right)-$ функиии из (1.8));

2) $\left|G_{0}(z)\right| \leqslant 1$ в $\mathbb{C}$;

3) $\left|c_{x}\left(G_{0}\right)\right| \geqslant A \sum_{s=1}^{n} d\left(R_{s}\right)$.

Заметим $\left[10 ;\right.$ п. 4], что функция $G_{0}(z)$, удовлетворяющая условию 1$)$ леммы 1.5 , имитирует своим поведением сингулярньй интеграл от ограниченной функции, заданной на липшицевой кривой $\Gamma^{\prime}$. Поэтому лемму 1.5 можно рассматривать как некоторьй дискретный аналог теоремы Нгуйена (Nguyen Xuan Uy) [15; теорема 3].

\section{§ 2. Схема доказательства основной леммы}

Здесь изложим доказательство леммы 1.4, не останавливаясь на обосновании вспомогательных утверждений (их доказательства вынесены в следующие параграфы). В $\S 3$ подробно рассматривается геометрическая конструкция (доказываются леммы 2.1-2.4), в $§ 4$ доказывается ключевая оценка (лемма 2.6).

Будем рассматривать замкнутые двоичные квадраты $Q_{k}^{n, l}$, определенные в $\S 1$. $\left(\right.$ Напомним, что $Q_{k}^{n, l}=\left[n 2^{-k},(n+1) 2^{-k}\right] \times\left[l 2^{-k},(l+1) 2^{-k}\right]$ при некоторых целых $k, n$ и $l$.) Заметим, что если пересечение двух таких квадратов содержит открытое множество, то один из них содержится в другом. Вертикальным рядом будем назьвать множество всех таких квадратов $Q_{k}^{n, l}$, для которых $k$ и $n$ фиксированы. Два вертикальных ряда $\left\{Q_{k}^{n_{1}, l}\right\}$ и $\left\{Q_{k}^{n_{2}, l}\right\}\left(k, n_{1}\right.$ и $n_{2}=$ const) назовем соседними, если $\left|n_{1}-n_{2}\right|=1$. 
Для дальнейшей конструкции важным является следующее определение. Два квадрата $Q_{k}^{n_{1}, l_{1}}$ и $Q_{k}^{n_{2}, l_{2}}$ (одного размера) назовем согласованньлми (согласованной nарой), если одновременно выполнены условия: $\left|n_{1}-n_{2}\right| \leqslant 1$ и $2 \leqslant\left|l_{1}-l_{2}\right| \leqslant 3$.

Говоря о покрытии некоторого компакта конечным множеством замкнутых двоичных квадратов, всегда будем считать, что выполнены два условия:

1) каждый квадрат покрытия имеет с компактом общие точки;

2) никакой из квадратов покрытия не содержится в другом.

Рассмотрим следующую геометрическую лемму 2.1. Эта лемма будет справедлива и в предположении, что $E$ - произвольньй непустой компакт, принадлежащий квадрату $Q_{0}$, не обязательно замыкание множества $\stackrel{\circ}{E}$ из леммы 1.4 .

Сделаем ряд пояснений. В лемме 2.1 расматриваются покрытия $\mathscr{Q}\left(k_{0}\right)$ компакта $E, k_{0}=0,1,2, \ldots$, состоящие из конечных множеств замкнутых двоичных квадратов и обладающие дополнительными свойствами 1$)-4)$. При этом $\mathscr{Q}(0)=Q_{0}$ и последовательность покрытий является "невозрастающей", т.е. каждый квадрат покрытия $\mathscr{Q}\left(k_{0}+1\right)$ содержится в некотором (соответствуюшем) квадрате из $\mathscr{Q}\left(k_{0}\right)$.

Множество квадратов каждого покрытия разбито на два непересекающихся класса - классы так называемых красных и бельх квадратов (исходный квадрат $Q_{0}$ белый). Каждый красный квадрат покрытия $\mathscr{Q}\left(k_{0}\right)$ на следующих шагах не изменяет ни своего цвета, ни размера и принадлежит всем покрытиям $\mathscr{Q}(k)$ при $k>k_{0}$. (Таким образом, если на каком-то шаге $k_{0}$ все квадраты покрытия становятся красными, то покрытие в дальнейшем не изменяется.) В противоположность этому каждый белый квадрат на следуюшем шаге разбивается на более мелкие (процедура разбиения и определения цвета полученных квадратов рассматривается в доказательстве леммы 2.1).

ЛЕмма 2.1 (геометрическая). Для любого $k_{0}=0,1,2, \ldots$ существуют покрытие $2\left(k_{0}\right)$ компакта $E$ семейством замкнутых двоичных квадратов и ломаная $\Gamma\left(k_{0}\right)$ такие, что выполняются следующие свойства 1)-4):

1) длина сторонь каждого белого квадрата равна $2^{-k_{0}}$, длина стороньь красного квадрата может принимать значение $2^{-k}, k=1,2, \ldots, k_{0}$;

2) пусть прямая, параллельная оси Оу, проходит через внутреннюю точку некоторого квадрата $Q$ из $2\left(k_{0}\right)$, тогда она может пересекать квадрать из $\mathscr{Q}\left(k_{0}\right)$ только того же цвета и размера, что и у $Q$, причем, в общей сложности, не более четырех красных (если $Q$ красный) или не более двух бельи, имеющих общую сторону (если $Q$ бельй);

3) если в вертикальном ряду найдется хотя бъ один красньий квадрат $Q$ из $2\left(k_{0}\right)$, то всегда существует согласованная пара красных квадратов из $2\left(k_{0}\right)$, один из которых содержится в том же вертикальном ряду, что и $Q$ (а другой в силу определения согласованных квадратов - в том жее вертикальном ряду или в соседнем);

4) ломаная $\Gamma=\Gamma\left(k_{0}\right)$ проводится через чентры некоторых квадратов из $2\left(k_{0}\right)$, причем в каждом вертикальном ряду используется не более одного квадрата. Если ломаная Г не вырождается в точку, то она является графиком некоторой функции $y=\psi(x)=\psi_{k_{0}}(x)$, для которой $\left|\psi^{\prime}(x)\right| \leqslant A$, при әтом для каждого квадрата $Q$ из $\mathscr{Q}\left(k_{0}\right)$ выполнено условие: $\operatorname{dist}(Q, \Gamma) \leqslant$ $\operatorname{Ad}(Q)$. 
Лемма 2.1 доказывается в $\S 3$.

Вернемся теперь к компакту $E$ из леммы 1.4 и, применяя лемму 2.1, выберем "момент остановки", т.е. зафиксируем подходяшее $k_{0}$ (а также $\mathscr{Q}=\mathscr{Q}\left(k_{0}\right)$ и $\Gamma=$ $\left.\Gamma\left(k_{0}\right)\right)$. Для этого используем регулярную мажсоранту модуля непрерывности $\oint y н к и и и ~ \Phi(z)$, т.е. такую функцию $\omega(\delta)$, для которой выполнены условия: $\omega(\delta) \geqslant$ $\omega_{\Phi}(\delta), \omega(2 \delta) \leqslant A \omega(\delta)$ и $\lim _{\delta \rightarrow 0+0} \omega(\delta)=0$.

В дальнейшей части доказательства леммы 1.4 считаем, что $k_{0}$ - минимальный номер, при котором вьполнено хотя бы одно из следующих двух условий:

1) семейство $\mathscr{Q}\left(k_{0}\right)$ состоит только из красных квадратов;

2) $\omega\left(2^{-k_{0}}\right)<\sum d_{\mathrm{Kp}}$,

где в правой части неравенства записана сумма $d(Q)$ по всем красным квадратам $Q$ покрытия $\mathscr{Q}\left(k_{0}\right)$.

Ясно, что указанный номер $k_{0}$ сушествует. Действительно, в противном случае, так как правая часть неравенства $(2.1)$ не убывает с ростом $k_{0}$, все покрытия $\mathscr{Q}\left(k_{0}\right)$ должны состоять только из белых квадратов. Но это невозможно из-за того, что в силу условий 1$)$ и 2$)$ леммы 2.1 сумма площадей белых квадратов из $\mathscr{Q}\left(k_{0}\right)$ стремится к нулю при $k_{0} \rightarrow \infty$, а покрываемый этими квадратами компакт $E$ является замыканием непустого открытого множества.

Теперь, зафиксировав $k_{0}$, уточним выбор точек $z_{\iota}$ леммы 1.4 . Для каждого квадрата $Q$ из покрытия $\mathscr{Q}$ (напомним, что $Q$ обязательно пересекает компакт $E$ ) выберем точку $z(Q) \in 1.1 Q \cap \stackrel{\circ}{E}$. Пусть $m$ - общее число выбранных точек; зафиксируем их и, упорядочив, обозначим $z_{\iota}, \iota=1, \ldots, m$. Так как в силу леммы $\left.2.1,3\right)$ и условий выбора $k_{0}$ покрытие $\mathscr{Q}$ содержит хотя бы одну пару согласованных красных квадратов, то обязательно $m>1$.

Так как функция $G(z)$ из условия 1$)$ леммы 1.4 является разрывной в квадрате $D_{0}=[-1,2] \times[-1,2]$, то будет удобно несколько уменьшить $D_{0}$, “вырезав" маленькие открытые круги с центрами в точках $z_{\iota}$. Пусть $B_{\iota}=B\left(z_{\iota}, \varepsilon_{\iota}\right)$ - попарно непересекающиеся круги, причем $B_{\iota} \in 1.1 Q_{0}, \iota=1, \ldots, m$. Рассмотрим компакт

$$
\widetilde{D}_{0}=D_{0} \backslash\left(\bigcup_{\iota=1}^{m} 0.5 \stackrel{\circ}{B_{\iota}}\right) \text {. }
$$

Лемма 1.2 позволяет заменить в лемме 1.4 условие 2$)$ на следуюшее: $|G(z)| \leqslant 1$ на $\widetilde{D}_{0}$ (и поэтому мы будем оценивать $|G(z)|$ на $\widetilde{D}_{0}$, а не на $D_{0}$ ).

Чтобы переформулировать лемму 1.4 в более удобном виде, введем следующие функциональные пространства. Пусть $C\left(\widetilde{D}_{0}\right)-$ пространство функиий $g(z)$, непрерывных на $\widetilde{D}_{0}$ с равномерной нормой: $\|g\|=\max _{\widetilde{D}_{0}}|g(z)|$. По теореме $\Phi$. Рисса сопряженным к $C\left(\widetilde{D}_{0}\right)$ является пространство комплексных борелевских мер с носителями на $\widetilde{D}_{0}$, в котором норма определяется как полная вариация меры: $\|\mu\|=\int_{\widetilde{D}_{0}}|d \mu|$.

Пусть $H_{0}\left(\widetilde{D}_{0}\right)$ - пространство функций следующего вида $\left(z \in \widetilde{D}_{0}\right)$ :

$$
G(z)=\sum_{\iota=1}^{m} \lambda_{\iota} \frac{\overline{z-z_{\iota}}}{z-z_{\iota}}, \quad \text { где } \sum_{\iota=1}^{m} \lambda_{\iota}=0
$$


которое будем рассматривать как $(m-1)$-мерное комплексное подпространство $C\left(\widetilde{D}_{0}\right)$.

Напомним, что в силу $\sum_{\iota=1}^{m} \lambda_{\iota}=0$ коэффицциент $c_{x}(G)$ не зависит от центра разложения функции $G$ в ряд Лорана. Пусть $\mu$ - комплексная борелевская мера $c \operatorname{supp} \mu \subset \widetilde{D}_{0}$ такая, что что для каждой функции $G(z)$ вида $(2.2)$ выполнено условие:

$$
c_{x}(G)=\int G(z) d \mu(z) .
$$

Тогда будем называть $\mu$ мерой, представляющей функиионал $c_{x}$ на пространстве $H_{0}\left(\widetilde{D}_{0}\right)$ (кратко - представляющей мерой).

Множество представляюших мер заведомо непусто. Применив формулу (1.3), нетрудно построить регулярную представляющую меру с носителем в $D_{0} \backslash 1.1 Q_{0}$ (точку $z_{0}$ в (1.3) можно взять произвольно).

Переформулируем лемму 1.4 в терминах представляющих мер. Нужно доказать, что

$$
\sup _{G \in H_{0}\left(\widetilde{D}_{0}\right),\|G\| \leqslant 1}\left|c_{x}(G)\right| \geqslant A_{1}\left|c_{x}(\Phi)\right| .
$$

По теореме Хана-Банаха, примененной к пространству $C\left(\widetilde{D}_{0}\right)$ и его подпространству $H_{0}\left(\widetilde{D}_{0}\right)$, это равносильно тому, что для любой фиксированной представляюшей меры $\tilde{\mu}$ верна оценка:

$$
\inf _{\mu_{0}}\left\|\tilde{\mu}+\mu_{0}\right\| \geqslant A_{1}\left|c_{x}(\Phi)\right|
$$

где нижняя грань берется по всем мерам $\mu_{0}$, ортогональньм $H_{0}\left(\widetilde{D}_{0}\right)$, т.е. таким, что для каждой функции $G(z)$ из $(2.2)$ выполнено условие: $\int G(z) d \mu_{0}(z)=0$.

Так как множество всех мер вида $\tilde{\mu}+\mu_{0}$ совпадает с множеством всех представляюших мер, то лемма 1.4 сводится к следующей оценке, которую мы и будем доказывать.

ЛЕмма $1.4^{\prime}$. Для любой представляющей меры $\mu$ выполнено условие:

$$
\|\mu\| \geqslant A_{1}\left|c_{x}(\Phi)\right|
$$

ДокАЗАТЕЛЬСТво ЛЕммы $1.4^{\prime}$. Прежде всего (в 33 устанавливается более грубая оценка:

Лемма 2.2. Существует функиия $G_{1}(z)$ вида $(2.2),\left|G_{1}(z)\right| \leqslant 1$ в $\mathbb{C}$, такая, что $\left|c_{x}\left(G_{1}\right)\right| \geqslant A \sum d_{\mathrm{Kp}}$, где сумма $\sum d_{\mathrm{Kp}}$ определена в $(2.1)$.

Лемма 2.2 является несложным следствием леммы 1.5, условия 3) леммы 2.1 и определения согласованных квадратов. (В силу этого определения для каждой согласованной пары красных квадратов $Q^{\prime}$ и $Q^{\prime \prime}$ со стороной $d$ найдется пара точек $z^{\prime}$ и $z^{\prime \prime}$ из (2.2), расположенных соответственно “вблизи" $Q^{\prime}$ и $Q^{\prime \prime}$ и таких, что $\left|\operatorname{Im}\left(z^{\prime}-z^{\prime \prime}\right)\right| \geqslant A_{1} d$.) Следуюшее утверждение очевидно. 
СЛЕДСТВИЕ ЛЕМмЫ 2.2. Для любой представляющей меры $\mu$ выполнена оченка: $\|\mu\| \geqslant A \sum d_{\mathrm{kp}}($ әде $A$ из леммы 2.2).

Заметим, что если выполнено условие $\sum d_{\text {кр }} \geqslant A\left|c_{x}(\Phi)\right|$, то в силу леммы 2.2 оценка (2.3) установлена, тем самым выполнение условия $\sum d_{\text {кр }} \geqslant A\left|c_{x}(\Phi)\right|$ также может быть взято в качестве одного из "моментов остановки" для выбора $k_{0}$. Однако это очень специальный частный случай леммы $1.4^{\prime}$. В действительности, $\left|c_{x}(\Phi)\right|$ может, вообще говоря, “значительно превысить" не только $\sum d_{\text {кр }}$, но даже $\sum d_{\mathrm{kp}}+\operatorname{Var}(\Gamma)$, что, по меньшей мере, сушественно затрудняет возможность явного построения функции $G(z)$ из леммы 1.4 .

С этого момента зафиксируем представляющую меру $\mu$. Нам известно (следствие леммы 2.2), что $\|\mu\| \geqslant A \sum d_{\text {кр }}$, кроме того, считаем, что $\|\mu\| \leqslant 1$ (в противном случае доказьвать нечего).

Прежде всего необходимо “скорректировать" покрытие $\mathscr{Q}$ из леммы 2.1 с учетом структуры представляюшей меры $\mu$. Вообше говоря, сушествуют такие квадраты $Q$ из $\mathscr{Q}$, что вариация порции меры $\mu$, расположенной “вблизи” $Q$, “значительно превышает" $d(Q)$. Поэтому некоторые квадраты из $\mathscr{Q}$ будут увеличены, что приведет к новому покрытию $\mathscr{Q}^{\prime}$ компакта $E$ семейством замкнутых двоичных квадратов. Цель нового покрытия - выполнение оценок леммы 2.5. Как следствие получается ключевая оценка леммы 2.6, из которой в этом параграфе и вьводится лемма $1.4^{\prime}$. Соответствующая процедура перехода к $\mathscr{Q}^{\prime}$ рассматривается в лем$\max 2.3$ и 2.4 .

В лемме 2.3 проведем для меры $\mu$ стандартное разложение Кальдерона-Зигмунда (аналогично, например, [13; гл. 1, п. 3.3]).

Напомним, что минимальная длина стороны квадратов из покрытия $\mathscr{Q}$ равна $2^{-k_{0}}$, a $D_{0}=[-1,2] \times[-1,2]$. Для произвольного квадрата $D$ под $\|\mu\|(D)$ будем понимать $\int_{D}|d \mu|$ (также $\mu(D)=\int_{D} d \mu$ ).

Лемма 2.3. Существует разложение квадрата $D_{0}$ на семейство замкнутых двоичных квадратов (так называемых "хороиих" и "плохих" квадратов, $D_{g}$ и $D_{b}$ соответственно) со следующими свойствами:

1) $d\left(D_{g}\right)=2^{-k_{0}}, d\left(D_{b}\right)$ можст принимать значения $2^{-k}, k=1,2, \ldots, k_{0}$;

2) пусть $D$ - произвольный замкнутый двоичный квадрат, содержащий хотя бы один хороший или плохой квадрат, тогда $\|\mu\|(D) \leqslant 4 d(D)$;

3) для любого хорошего квадрата выполнена оценка: $\|\mu\|\left(D_{g}\right) \leqslant d\left(D_{g}\right)$, a для любого плохого, соответственно, двусторонняя оценка:

$$
d\left(D_{b}\right)<\|\mu\|\left(D_{b}\right) \leqslant 4 d\left(D_{b}\right)
$$

4) сумма $d\left(D_{b}\right)$, взятая по всем плохим квадратам, не превосходит $4\|\mu\|$.

Лемма 2.3 доказывается в $\S 3$.

Теперь, используя лемму 2.3, увеличим некоторые квадраты из $\mathscr{Q}$ по включению (это означает, что квадрат $Q$ заменяется на некоторый замкнутьй двоичный квадрат $Q^{\prime}$ такой, что $\left.Q \subset Q^{\prime}\right)$. Затем в полученном семействе оставим квадраты, максимальные по включению, и тем самьм придем к покрытию $\mathscr{Q}^{\prime}$ из следующей леммы 2.4, которая доказывается в $\S 3$. 
Лемма 2.4. Существует покрытие $\mathscr{Q}^{\prime}$ компакта Е семейством замкнутых двоичных көадратов. Семейство разбито на два непересекающихся класса: классы новых бельх и новых красных квадратов (в дальнейшем слово “новые” будем опускать). Покрытие обладает следующими свойствами:

1) длина стороны каждого белого квадрата равна $2^{-k_{0}}$, длина сторонь каждого красного квадрата моэсет принимать значение $2^{-k}, k=1,2, \ldots, k_{0}$; при этом выполнено условие:

$$
\sum^{\prime} d_{\mathrm{\kappa p}} \leqslant A\|\mu\|, \quad \text { əде } \quad \sum^{\prime} d_{\mathrm{Kp}}=\sum_{\{Q \mid Q(\text { новый }) \text { красный }\}} d(Q) \text {; }
$$

2) пусть $Q$ - произвольный квадрат покрытия $\mathscr{Q}^{\prime}, D$ - хороиий или плохой квадрат из леммы 2.3. Тогда выполняется следующее условие: если $Q$ и $D$ пересекаются (хотя бы в одной точке), то $d(Q) \geqslant d(D)$; если жсе указанные квадраты не пересекаются, то $\operatorname{dist}(Q, D) \geqslant d(D)$;

3) для каждого квадрата $Q$ из покрытия $\mathscr{Q}^{\prime}$ выполнено условие:

$$
\operatorname{dist}(Q, \Gamma) \leqslant A d(Q)
$$

где Г - ломаная из леммы 2.1,4);

4) пусть прямая, параллельная оси $O y$, проходит через внутреннюю точку некоторого квадрата $Q$ из $\mathscr{Q}^{\prime}$; тогда она может пересекать квадратьи из $\mathscr{Q}^{\prime}$ только того жсе ивета и размера, что и у $Q$, причем число таких квадратов не превосходит $A_{1}$;

5) для каждого квадрата $Q$ из $\mathscr{Q}^{\prime}$ найдется какая-либо из точек $z_{\iota}$ (формульи (2.2)) такая, что $z_{\iota} \in 1.1 Q \cap \stackrel{\circ}{E}$.

Заметим, что условие 4) леммы 2.4 оказывается несколько ослабленным по сравнению с соответствующим условием 2) леммы 2.1 .

Из лемм 2.2-2.4 и условия (2.1) выбора $k_{0}$ немедленно вытекают следующие оценки, нужные для доказательства лемм 2.6 и $1.4^{\prime}$.

Лемма 2.5. 1) Пусть $Q$ - произвольный квадрат из покрытия $\mathscr{Q}^{\prime}$, а $B$ круг радиуса $r$, содерәсащий $Q$. Тогда $\|\mu\|(B) \leqslant A r$.

2) $\omega\left(2^{-k_{0}}\right) \sum^{\prime} d_{\text {бел }}+\sum^{\prime} d_{\mathrm{кр}} \leqslant A\|\mu\|$, где $\sum^{\prime} d_{\text {бел }} u \sum^{\prime} d_{\text {кр }}-$ суммы $d(Q)$ соответственно по всем белым и красным квадратам покрытия $\mathscr{Q}^{\prime}$.

ДоказАТЕльство Леммы 2.5. Докажем оценку 1). Пусть $D$ и $D^{\prime} \neq D$ - замкнутые двоичные квадраты одного размера такие, что $D$ содержит $Q$, а $D^{\prime}$ имеет с $D$ обшую граничную точку. Тогда в силу условия 2) леммы 2.4 квадрат $D$ обязательно содержит хороший или плохой квадрат из леммы 2.3 и, следовательно, в силу условия 2) леммы 2.3 выполняется оценка: $\|\mu\|(D) \leqslant 4 d(D)$. Оценка $\|\mu\|\left(D^{\prime}\right) \leqslant 4 d(D)$ доказывается точно так же. Действительно, если найдется квадрат $D^{\prime \prime}$ из леммы 2.3 , для которого $D^{\prime}$ является собственной частью (и, значит, $\left.d\left(D^{\prime \prime}\right) \geqslant 2 d\left(D^{\prime}\right)\right)$, то $\operatorname{dist}\left(Q, D^{\prime \prime}\right)<d\left(D^{\prime \prime}\right)$, что противоречит условию 2$)$ леммы 2.4 (и, следовательно, $D^{\prime}$ также содержит хороший или плохой квадрат).

Таким образом, $\|\mu\|(3 D) \leqslant 36 d(D)$, откуда сразу же следует нужная оценка и для круга $B$ радиуса $r$, “соизмеримого" с $d(D)$. 
Чтобы доказать 2), заметим, что в силу условия 1) леммы $2.4 \sum^{\prime} d_{\text {кр }} \leqslant A_{1}\|\mu\|$; из леммы $2.4,4)$ следует: $\sum^{\prime} d_{\text {бел }} \leqslant A_{1} ;$ из $(2.1)$ вытекает: $\omega\left(2^{-k_{0}}\right) \leqslant \sum d_{\text {кр }}$, а из леммы $2.2-\sum d_{\mathrm{kp}} \leqslant A_{1}\|\mu\|$. Лемма 2.5 доказана.

Поясним смысл оценок леммы 2.5. Если бы для любого круга $B=B\left(z_{0}, r\right)$ выполнялось условие $\|\mu\|(B) \leqslant A r$, то в покрытии $\mathscr{Q}^{\prime}$ не было бы необходимости: для доказательства леммы 2.6 (и, следовательно, леммы $1.4^{\prime}$ ) использовалось бы покрытие Q. Оценки 1) и 2) леммы 2.5 представляют собой некоторый компромисс. Увеличение квадратов покрытия является "вынужденной платой" за выполнение оценки 1) - дискретного аналога условия $\|\mu\|(B) \leqslant A r$. При этом оценка 2$)$ показывает необходимую степень увеличения квадратов.

Покрытие $\mathscr{Q}^{\prime}$ является основой для разбиения единицы на $E$ и локализации особеностей функции $\Phi(z)$.

Пусть $Q_{j}, j=1,2, \ldots,-$ все квадраты покрытия $\mathscr{Q}^{\prime}$. Применив лемму Харви и Полкинга [16; лемма 3.1$]$, построим семейство функций $\left\{\varphi_{j}\right\}$ таких, что для каждого номера $j$ функция $\varphi_{j}(z)$ стандартна относительно соответствуюшего квадрата $1.5 Q_{j}$ и при этом $\sum_{j} \varphi_{j} \equiv 1$ в некоторой окрестности $E$.

Указанные функции строятся по индукции [16]. Упорядочим квадраты $Q_{j}$ следуюшим образом: $d\left(Q_{1}\right) \geqslant \cdots \geqslant d\left(Q_{j}\right) \geqslant d\left(Q_{j+1}\right) \geqslant \cdots$. Пусть для любого $j$ $\widetilde{\varphi_{j}(z)}$ - функция, стандартная относительно $1.5 Q_{j}$, такая, что $\widetilde{\varphi_{j}} \equiv 1$ на $1.1 Q_{j}$.

Возьмем $\varphi_{1}=\widetilde{\varphi_{1}}$, а для $j \geqslant 2$ положим: $\varphi_{j}=\widetilde{\varphi_{j}} \prod_{p=1}^{j-1}\left(1-\widetilde{\varphi_{p}}\right)$. При этом, как нетрудно проверить по индукции, $\sum_{j} \varphi_{j}=1-\prod_{j}\left(1-\widetilde{\varphi_{j}}\right)$, а нужные оценки производных $\left(\left|\nabla^{l} \varphi_{j}(z)\right| \leqslant A\left(d\left(Q_{j}\right)\right)^{-l}, l=0,1,2\right)$ вытекают из того, что $d\left(Q_{j}\right)$ не возрастают с увеличением $j$.

Используя построенное разбиение единицы, локализуем особенности функции $\Phi(z)$. Из (1.1) следует, что

$$
\Phi(z)=\sum_{j} \Phi_{j}(z), \quad \text { где } \quad \Phi_{j}(z)=\pi^{-1} \frac{\bar{z}}{z} *\left(\varphi_{j} \bar{\partial}^{2} \Phi\right) .
$$

При этом в силу леммы $2.4,5)$ для каждого $j$ найдется точка $z_{j} \in 1.1 Q_{j} \cap \stackrel{\circ}{E}$ из (2.2). Рассмотрим функции:

$\Psi_{j}(z)=\Phi_{j}(z)-c_{0}\left(\Phi_{j}\right) \frac{\overline{z-z_{j}}}{z-z_{j}}, \quad G_{2}(z)=\sum_{j} c_{0}\left(\Phi_{j}\right) \frac{\overline{z-z_{j}}}{z-z_{j}}, \quad \Psi(z)=\sum_{j} \Psi_{j}(z)$.

Следуюшая лемма доказьвается в $\S 4$. Идея ее доказательства состоит в том, что функция $\Psi(z)$ имитирует своим поведением сингулярный интеграл от ограниченной функции, заданной на липшицевой кривой, а для меры $\mu$ выполнен "дискретный аналог карлесоновости": условие 1) леммы 2.5.

ЛЕмма 2.6 (ключевая оценка). Для функиии $\Psi(z)$ из (2.4) въполнена оцен$\kappa a$ :

$$
\left|\int \Psi(z) d \mu(z)\right| \leqslant A\|\mu\| \text {. }
$$

Используя лемму 2.6, завершим доказательство леммы 1.4' (и, следовательно, леммы 1.4). 
Заметим, что $\lim _{z \rightarrow \infty} \Psi_{j}(z)=0$. При этом из леммы 1.1 следует:

$$
\max \left(\left|c_{x}\left(\Psi_{j}\right)\right|,\left|c_{y}\left(\Psi_{j}\right)\right|\right) \leqslant \begin{cases}A d\left(Q_{j}\right), & \text { если } Q_{j} \text { красный, } \\ A \omega\left(2^{-k_{0}}\right) d\left(Q_{j}\right), & \text { если } Q_{j} \text { белый. }\end{cases}
$$

Оценим $\left|c_{x}(\Psi)\right|$, используя лемму $\left.2.5,2\right)$ и $(2.5)$. Получим:

$$
\left|c_{x}(\Psi)\right| \leqslant \sum_{j}\left|c_{x}\left(\Psi_{j}\right)\right| \leqslant A\left(\omega\left(2^{-k_{0}}\right) \sum^{\prime} d_{\text {бел }}+\sum^{\prime} d_{\mathrm{Kp}}\right) \leqslant A_{1}\|\mu\| .
$$

Заметим, что в силу $c_{0}(\Phi)=\sum_{j} c_{0}\left(\Phi_{j}\right)=0$ функция $G_{2}(z)$ из $(2.4)$ принадлежит пространству $H_{0}\left(\widetilde{D}_{0}\right)$. Так как мера $\mu$ представляет функционал $c_{x}$ на этом пространстве, то

$$
\int G_{2}(z) d \mu(z)=c_{x}\left(G_{2}\right)=c_{x}(\Phi)-c_{x}(\Psi)
$$

Отсюда и из (2.4) вытекает тождество:

$$
\int \Phi(z) d \mu(z)=\int \Psi(z) d \mu(z)+c_{x}(\Phi)-c_{x}(\Psi)
$$

Так как $|\Phi(z)| \leqslant 1$, то $\left|\int \Phi(z) d \mu(z)\right| \leqslant\|\mu\|$. В силу (2.6) $\left|c_{x}(\Psi)\right| \leqslant A\|\mu\|$, а в силу леммы $2.6\left|\int \Psi(z) d \mu(z)\right| \leqslant A\|\mu\|$.

Таким образом, $\|\mu\| \geqslant A_{1}\left|c_{x}(\Phi)\right|$. Доказательство леммы $1.4^{\prime}$ завершено.

\section{§3. Конструкция}

Леммы 2.1-2.4 в совокупности представляют собой геометрическую конструкцию леммы 1.4. В этом параграфе они доказываются.

ДокАЗАТЕЛЬСТво ЛЕммы 2.1. В доказательстве под квадратами всегда будем понимать замкнутые двоичные квадраты. Покрытия $\mathscr{Q}\left(k_{0}\right)$ строим индукцией по $k_{0}$.

Построение $2(1)$. Разобьем исходный бельй квадрат $Q_{0}$ на четыре равных квадрата (со стороной 0.5). Включим в $\mathscr{Q}(1)$ только те из них, которые пересекают компакт $E$, и назовем их бельмми.

“Наследуемые" свойства покрытия $\mathscr{Q}\left(k_{0}\right)$. Покрытие $\mathscr{Q}\left(k_{0}\right)$ состоит из множества (возможно, пустого) красных квадратов предыдушего покрытия $\mathscr{Q}\left(k_{0}-1\right)$, сторона которых превышает $2^{-k_{0}}$, а также из красных и белых квадратов со стороной $2^{-k_{0}}$, множество которых будем называть поколением порядка $k_{0}$. При этом для белых квадратов $Q^{n, l}=Q_{k_{0}}^{n, l}$ (поколения $k_{0}$ ) выполнено следуюшее условие. $E c л u$ для каких-либо двух из них: $Q^{n_{1}, l_{1}}$ и $Q^{n_{2}, l_{2}}$, имеет место $\left|n_{1}-n_{2}\right| \leqslant 1$, то обязательно $\left|l_{1}-l_{2}\right| \leqslant 1$ (в частности, нет ни одной пары согласованных бельх квадратов). 
Индукционный переход: построение $\mathscr{Q}\left(k_{0}+1\right)$. Разобьем каждый белый квадрат покрытия $\mathscr{Q}\left(k_{0}\right)$ на четыре равных квадрата (при этом породившиий их квадрат из рассмотрения исключается). Из полученных квадратов оставляем только те, которые пересекают $E$ (это будут квадраты $\left(k_{0}+1\right)$-го поколения). Именно они вместе с красньми квадратами покрытия $\mathscr{Q}\left(k_{0}\right)$ и составят $\mathscr{Q}\left(k_{0}+1\right)$. Осталось определить цвет квадратов $\left(k_{0}+1\right)$-го поколения. Сначала назовем их белыми, а затем проведем процедуру переименования. Будем продолжать эту процедуру до тех пор, пока останется хотя бы одна согласованная пара белых квадратов.

Процедура переименования. Пусть $Q_{k_{0}+1}^{n_{1}, l_{1}}, Q_{k_{0}+1}^{n_{2}, l_{2}}$ - какая-либо согласованная пара белых квадратов. Тогда, изменив цвет, назовем каждый бельй квадрат $Q_{k_{0}+1}^{n, l}$

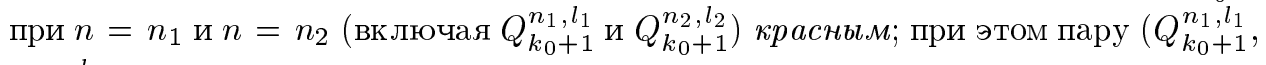
$Q_{k_{0}+1}^{n_{2}, l_{2}}$ ) также назовем отмеченной парой. После этого вернемся к поиску оставшихся согласованных пар белых квадратов.

Окончание указанной прочедуры завершает построение покрытия $\mathscr{Q}\left(k_{0}+1\right)$.

Чтобы проверить, что покрытие $\mathscr{Q}\left(k_{0}+1\right)$ "наследует свойства" $\mathscr{Q}\left(k_{0}\right)$, надо убедиться в следующем. Пусть $Q_{k_{0}+1}^{n_{1}, l_{1}}$ и $Q_{k_{0}+1}^{n_{2}, l_{2}}$ - какие-либо белые квадраты, для которых $\left|n_{1}-n_{2}\right| \leqslant 1$. Тогда (как и для белых квадратов $\mathscr{Q}\left(k_{0}\right)$ ) выполнено условие: $\left|l_{1}-l_{2}\right| \leqslant 1$. Действительно, сразу после разбиения на четыре части белых квадратов $k_{0}$-го поколения имело место: $\left|l_{1}-l_{2}\right| \leqslant 3$, а по окончании процедуры переименования, за счет устранения согласованных пар белых квадратов, получим $\left|l_{1}-l_{2}\right| \leqslant 1$.

Заметим, что из построения вытекает следуюший факт. Для любых двух квадратов $Q_{k}^{n_{1}, l_{1}}$ и $Q_{k}^{n_{2}, l_{2}}$ (одного размера) из покрытия $\mathscr{Q}(k)$, находящихся в одном вертикальном ряду или в соседних, вьполнено условие:

$$
\left|l_{1}-l_{2}\right| \leqslant 3
$$

(причем если указаннье квадратьи белье, то $\left|l_{1}-l_{2}\right| \leqslant 1$ ).

Зафикиируем номер $k_{0}$ и проверим вьполнение свойств 1)-4). Свойство 1) очевидно. Свойство 3) следует из процедуры переименования; согласованная пара красных квадратов, о которой идет речь в свойстве, - отмеченная пара.

Докажем свойство 2). Пусть прямая, параллельная оси $O y$, проходит через внутреннюю точку какого-либо красного квадрата $Q$. Напомним, что при переименовании все квадраты того же вертикального ряда, что и у $Q$, пересекающие компакт $E$, получают красный цвет и в дальнейшем не изменяются. Поэтому указанная прямая из квадратов покрытия может пересекать только красные, причем одного размера. В силу (3.1) таких квадратов не более четырех. Пусть теперь прямая, параллельная оси $O y$, проходит через внутреннюю точку белого квадрата. Так как белые квадраты имеют минимальньй размер среди всех квадратов покрытия, то указанная прямая, пересекая красный квадрат, обязательно бы проходила через его внутреннюю точку. Свойство 2) доказано.

Докажем свойство 4). В каждом вертикальном ряду выберем произвольно по одному квадрату из $\mathscr{Q}\left(k_{0}\right)$ (если такие квадраты есть). Проведем ломаную $Г$ : $y=\psi(x)$ через центры выбранных квадратов (последовательно, по возрастанию $x)$. Как ясно из $(3.1)$, для каждого квадрата $Q$ из семейства $\mathscr{Q}\left(k_{0}\right)$ выполнено: $\operatorname{dist}(Q, \Gamma) \leqslant 2.5 d(Q)$. 
Пусть $Q^{1}$ и $Q^{2}$ - квадраты $\mathscr{Q}\left(k_{0}\right)$ из различных вертикальных рядов с центрами соответственно $\left(x_{1}, y_{1}\right)$ и $\left(x_{2}, y_{2}\right)$. Чтобы доказать, что $\left|\psi^{\prime}(x)\right| \leqslant A$, надо оценить сверху отношение $\left|y_{2}-y_{1}\right| /\left|x_{2}-x_{1}\right|$. Пусть $k, k \leqslant k_{0},-$ максимальный номер, при котором квадраты $Q^{1 v}=Q_{k}^{n_{1}, l_{1}}$ и $Q^{2 v}=Q_{k}^{n_{1}+1, l_{2}}$ из покрытия $\mathscr{Q}(k)$, содержашие соответственно $Q^{1}$ и $Q^{2}$, находятся в соседних вертикальных рядах. Тогда в силу (3.1) для $Q^{1 v}$ и $Q^{2 v}$ выполнено условие: $\left|l_{1}-l_{2}\right| \leqslant 3$.

Для определенности считаем, что $d\left(Q^{1}\right) \geqslant d\left(Q^{2}\right)$. При этом либо $Q^{1}=Q^{1 v}$ (и тогда, как нетрудно проверить, $\left|y_{2}-y_{1}\right| /\left|x_{2}-x_{1}\right| \leqslant 3.5 / 0.5=7$ ), либо в покрытии $\mathscr{Q}(k)$ квадраты $Q^{1 v}$ и $Q^{2 v}$ - белые, а два квадрата $(k+1)$-го поколения, содержащие соответственно $Q^{1}$ и $Q^{2}$, уже не находятся в соседних вертикальных рядах (и тогда $\left|y_{2}-y_{1}\right| /\left|x_{2}-x_{1}\right| \leqslant 2 / 0.5=4$ ). Таким образом, $\left|\psi^{\prime}(x)\right| \leqslant 7$. Свойство 4) доказано. Доказательство леммы 2.1 завершено.

ДокАЗАТЕльство леммы 2.2. Сведем лемму 2.2 к лемме 1.5 , где все $z_{s}$ и $z_{s}^{\prime}-$ некоторые из точек $z_{\iota}$ формулы (2.2). Соответствуюшее рассуждение аналогично проведенному в [10; п. 3]. С помощью этого технического рассуждения ломаная Г и отмеченные пары согласованных квадратов из доказательства леммы 2.1 заменяются на более удобные объекты: ломаную $\Gamma^{\prime}$ со значительно меньшей постоянной Липшица и "хорошо изолированные друг от друга" квадраты $R_{s}$ с центрами в вершинах $\Gamma^{\prime}$, при этом выбранные пары точек из $(2.2)$ лежат строго внутри квадратов $R_{s}$.

Пусть $\left(Q^{\prime}, Q^{\prime \prime}\right)$ - произвольная отмеченная пара согласованных красных квадратов, $z^{\prime} \in 1.1 Q^{\prime} \cap \stackrel{\circ}{E}$ и $z^{\prime \prime} \in 1.1 Q^{\prime \prime} \cap \stackrel{\circ}{E}-$ соответствующие ей точки из $(2.2)$. Из определения согласованных квадратов следует, что:

$$
\left|\operatorname{Im}\left(z^{\prime}-z^{\prime \prime}\right)\right| \geqslant \frac{9}{10} d\left(Q^{\prime}\right), \quad \frac{\left|\operatorname{Im}\left(z^{\prime}-z^{\prime \prime}\right)\right|}{\left|\operatorname{Re}\left(z^{\prime}-z^{\prime \prime}\right)\right|}>\frac{1}{4} .
$$

Для каждой пары точек $\left(z^{\prime}, z^{\prime \prime}\right)$ рассмотрим $R^{\prime}$ - замкнутый квадрат с центром в середине отрезка $\left[z^{\prime}, z^{\prime \prime}\right]$ и длиной стороны $d\left(R^{\prime}\right)=20\left|\operatorname{Im}\left(z^{\prime}-z^{\prime \prime}\right)\right|$. Как нетрудно убедиться, квадрат $R^{\prime}$ накрывает множество $Q^{\prime} \cup Q^{\prime \prime}$.

Пусть $Z^{\prime}$ - множество всех точек $z^{\prime}$ и $z^{\prime \prime}$, взятых по всем парам $\left(Q^{\prime}, Q^{\prime \prime}\right)$. Построим следуюшее множество квадратов $\mathscr{R}^{\prime \prime}$. Для этого рассмотрим всевозможные пары точек $z$ и $w$ из множества $Z^{\prime}$ таких, что $|\operatorname{Im}(z-w)| /|\operatorname{Re}(z-w)|>1 / 4$ (в частности, но не обязательно соответствующие парам согласованных квадратов). Для каждой пары точек $z$ и $w$ (как и вьше для $z^{\prime}$ и $z^{\prime \prime}$ ) построим квадрат с центром в середине отрезка $[z, w]$ и длиной стороны, равной $20|\operatorname{Im}(z-w)|$. Затем из построенных квадратов включим в $\mathscr{R}^{\prime \prime}$ только каждый такой квадрат, проекция которого на ось $O x$ не накрьвается соответствующей проекцией никакого другого.

Пусть $\Delta-$ множество точек оси $O x$, полученное при проектировании всех квадратов из $\mathscr{R}^{\prime \prime}$, а $|\Delta|-$ длина $\Delta$. В силу построения и леммы $\left.2.1,2\right)$ ясно, что $|\Delta| \geqslant$ $(1 / 4) \sum d_{\mathrm{kp}}$.

Теперь, применив лемму о покрытии (типа Витали) [13; гл. 1, п. 1.6], выберем только такие квадраты из $\mathscr{R}^{\prime \prime}$, проекции которых на ось $O x$ попарно не пересекаются и при этом сумма длин всех таких проекций не меншше $(1 / 5)|\Delta|$ и, следовательно, не меньше, чем $(1 / 20) \sum d_{\text {кр }}$. Напомним $[13 ;$ гл. 1, п. 1.7$]$, что для этого на каждом шаге выбирается наибольший квадрат из $\mathscr{R}^{\prime \prime}$ такой, что его проекция на ось $O x$ не 
пересекает проекции квадратов, выбранных ранее, - до тех пор, пока такой выбор возможен.)

Множество выбранных квадратов обозначим через $\mathscr{R}^{\prime}$, и пусть $n$ - число элементов $\mathscr{R}^{\prime}$. Обозначим эти квадраты $R_{1}^{\prime}, \ldots, R_{n}^{\prime}$, при этом упорядочим их так, чтобы $\operatorname{Re} t_{1}<\cdots<\operatorname{Re} t_{s}<\operatorname{Re} t_{s+1}<\cdots<\operatorname{Re} t_{n}$, где $t_{s}-$ центры квадратов $R_{s}^{\prime}$. Напомним, что $d\left(R_{s}^{\prime}\right)=20\left|\operatorname{Im}\left(z_{s}^{\prime}-z_{s}\right)\right|$, где $\left(z_{s}, z_{s}^{\prime}\right)$ - пара точек из $Z^{\prime}$, по которой был построен квадрат $R_{s}^{\prime},\left|\operatorname{Im}\left(z_{s}^{\prime}-z_{s}\right)\right| /\left|\operatorname{Re}\left(z_{s}^{\prime}-z_{s}\right)\right|>1 / 4, t_{s}$ - середина отрезка $\left[z_{s}, z_{s}^{\prime}\right]$ и $\sum_{s=1}^{n} d\left(R_{s}^{\prime}\right) \geqslant A \sum d_{\text {кр }}$. При этом проекции квадратов из $\mathscr{R}^{\prime}$ на ось $O x$ попарно не пересекаются.

Возьмем $R_{s}=(1 / 4) R_{s}^{\prime}, s=1, \ldots, n$. Оказывается, $R_{s}$ - требуемые квадраты леммы 1.5. Ясно, что $(1 / 5) d\left(R_{s}\right)=\left|\operatorname{Im}\left(z_{s}^{\prime}-z_{s}\right)\right|, z_{s}$ и $z_{s}^{\prime} \in R_{s}$, а $4 R_{s}$ попарно не пересекаются. Единственное, что нуждается в проверке - условие $\left|Y^{\prime}(x)\right| \leqslant 1 / 4$, где $y=Y(x)$ - уравнение ломаной $\Gamma^{\prime}$, проходящей последовательно через точки $t_{s}$, $s=1, \ldots, n$.

Действительно, в противном случае найдутся квадраты $R_{s_{1}}^{\prime}$ и $R_{s_{2}}^{\prime}$ из множества $\mathscr{R}^{\prime}$ с центрами $t_{s_{1}}$ и $t_{s_{2}}$ такими, что $\left|\operatorname{Im}\left(t_{s_{1}}-t_{s_{2}}\right)\right| /\left|\operatorname{Re}\left(t_{s_{1}}-t_{s_{2}}\right)\right|>1 / 4$. Но тогда найдутся следуюшие точки $w_{1}$ и $w_{2}: w_{1}=x_{1}+i y_{1}-$ какая-либо из точек $z_{s_{1}}$ или $z_{s_{1}}^{\prime}$, а $w_{2}=x_{2}+i y_{2}-$ какая-либо из точек $z_{s_{2}}$ или $z_{s_{2}}^{\prime}$, такие, что

$$
\frac{\left|y_{2}-y_{1}\right|}{\left|x_{2}-x_{1}\right|}>\frac{1}{4} \text {. }
$$

Покажем, что это невозможно. Заметим, что $\left|\operatorname{Re}\left(t_{s_{1}}\right)-x_{1}\right|<0.1 d\left(R_{s_{1}}^{\prime}\right)$, а также $\left|\operatorname{Re}\left(t_{s_{2}}\right)-x_{2}\right|<0.1 d\left(R_{s_{2}}^{\prime}\right)$. Поэтому, учитывая, что проекции квадратов из $\mathscr{R}^{\prime}$ на ось $O x$ попарно не пересекаются, получим: $\left|x_{2}-x_{1}\right|>0.4\left(d\left(R_{s_{1}}^{\prime}\right)+d\left(R_{s_{2}}^{\prime}\right)\right)$. Иначе говоря, точки $w_{1}$ и $w_{2}$ расположены "достаточно далеко друг от друга".

Отсюда следует, что в случае $\left|y_{2}-y_{1}\right| /\left|x_{2}-x_{1}\right|>1 / 4$ проекции на ось $O x$ квадратов $R_{s_{1}}^{\prime}$ и $R_{s_{2}}^{\prime}$ накрываются проекцией некоторого квадрата $R^{\prime}$, построенного тем же способом, что и выше, для указанной пары точек $w_{1}$ и $w_{2}$ из $Z^{\prime}$. Действительно, сторона $R^{\prime}$ “достаточно велика” по сравнению с $d\left(R_{s_{1}}^{\prime}\right), d\left(R_{s_{2}}^{\prime}\right)$ и $\left|x_{2}-x_{1}\right|$ :

$$
d\left(R^{\prime}\right)=20\left|y_{2}-y_{1}\right|>5\left|x_{2}-x_{1}\right|>d\left(R_{s_{1}}^{\prime}\right)+d\left(R_{s_{2}}^{\prime}\right)+2.5\left|x_{2}-x_{1}\right| .
$$

Таким образом, квадраты $R_{s_{1}}^{\prime}$ и $R_{s_{2}}^{\prime}$ не могли быть включены в множество $\mathscr{R}^{\prime \prime}$ и, следовательно, в $\mathscr{R}^{\prime}$.

Условия леммы 1.5 выполнены, и искомая функция $G_{1}(z)$ - в точности функция $G_{0}(z)$ из леммы 1.5. Лемма доказана.

ДокАЗАТЕЛьство леммы 2.3. Так же как и в доказательстве леммы 2.1, говоря о квадратах, будем иметь в виду замкнутые двоичные квадраты. Указанное семейство квадратов строим по индукции. Исходное (нулевое) поколение - девять квадратов со стороной равной $1\left(Q_{0}^{n, l}\right.$ при $\left.n, l=-1,0,1\right)$, составляющих $D_{0}$. Так как $\|\mu\| \leqslant 1$, эти квадраты хорошие. Источниками следующих поколений являются только хорошие квадраты, плохие фиксируются. На каждом следуюшем шаге каждый хороший квадрат делится на четыре равных квадрата. Если для какого-нибудь из них выполнено условие: $\|\mu\|(D)>d(D)$, назовем его плохим; в противном случае назовем хорошим. Процесс завершается по окончании построения $\left(k_{0}\right.$-го) поколения квадратов со стороной $2^{-k_{0}}$. 
Выполнение свойств 1) и 3) следует из построения. Докажем 2). Очевидно, достаточно считать, что $d(D)<1$ и $D \subset D_{0}$. Рассмотрим цепочку вложенных квадратов, соединяющую $D$ с соответствующим квадратом нулевого поколения. Так как плохие квадраты фиксируются, в этой цепочке в процессе построения все квадраты были хорошими, кроме, возможно, $D$ (и, следовательно, $\|\mu\|(D) \leqslant 4 d(D)$ ). Свойство 4) получается суммированием из условия $\|\mu\|\left(D_{b}\right)>d\left(D_{b}\right)$ и из того, что в одной точке пересекаются не более четырех квадратов. Лемма доказана.

ДоКАЗАТЕЛЬСТво ЛЕммы 2.4. Прежде всего построим вспомогательное покрытие $\mathscr{Q}^{\prime \prime}$, обладающее всеми свойствами, указанными в лемме 2.4 , за исключением, возможно, свойства 4 ). Затем, несколько изменив $\mathscr{Q}^{\prime \prime}$, придем к требуемому покрытию $\mathscr{Q}^{\prime}$.

Для каждого плохого квадрата $D_{b}$ из леммы 2.3 рассмотрим его "оболочку" $S\left(D_{b}\right)$ - множество, состояшее из девяти квадратов: самого квадрата $D_{b}$ и восьми окружающих его замкнутых двоичных квадратов того же размера, что и $D_{b}$. Построим множество $S^{\prime}$, состоящее из всех квадратов, принадлежащих $S\left(D_{b}\right)$ при различных $D_{b}$, а затем в $S^{\prime}$ возьмем максимальные квадраты по включению. Полученное множество обозначим через $S$. Как следует из леммы $2.3,4)$, сумма $d(Q)$ по всем квадратам $Q$ множества $S$ не превосходит $36\|\mu\|$.

Пусть $Q$ - произвольньй квадрат покрытия $\mathscr{Q}$. Если найдется квадрат $Q^{\prime \prime} \in S$ такой, что $Q$ является собственной частью $Q^{\prime \prime}$, то изменим $Q$, положив $Q=Q^{\prime \prime}$, и, при необходимости переименовав, будем считать $Q$ красным. Тем самьг, получим новое покрытие $\mathscr{Q}^{\prime \prime}$ компакта $E$ семейством (белых и красных) замкнутых двоичных квадратов.

Как следует из построения, для покрытия $\mathscr{Q}^{\prime \prime}$ выполняются: свойство 1) из леммы 2.4 (напомним, что по сравнению с покрытием $\mathscr{Q}$ сумма $d(Q)$ по всем красным квадратам $Q$ увеличилась не более чем на $36\|\mu\|$ ), а также свойство 2) (именно для выполнения которого строились оболочки $\left.S\left(D_{b}\right)\right)$. Свойства 3 ) и 5 ) для покрытия $\mathscr{Q}^{\prime \prime}$ выполняются потому, что они были справедливы для исходного покрытия $\mathscr{Q}$, а каждый квадрат из $\mathscr{Q}$ содержится в некотором (соответствующем) квадрате из $\mathscr{Q}^{\prime \prime}$.

Чтобы прийти к требуемому покрытию $\mathscr{Q}^{\prime}$, увеличим некоторые квадраты из $\mathscr{Q}^{\prime \prime}$ по включению. Очевидно, при этом сохранятся свойства 3) и 5), а также свойство 2) (если для квадрата $Q$ из $\mathscr{Q}^{\prime \prime}$ вьполняется условие $\operatorname{dist}\left(Q, D_{b}\right) \geqslant d\left(D_{b}\right)$, а для увеличенного квадрата $Q^{\prime}$ уже имеем: $\operatorname{dist}\left(Q^{\prime}, D_{b}\right)<d\left(D_{b}\right)$, то это возможно лишь в том случае, когда некоторый квадрат из $S\left(D_{b}\right)$ является собственной частью $Q^{\prime}$ и, следовательно, $\left.d\left(Q^{\prime}\right)>d(D)\right)$. Таким образом, в доказательстве будут нуждаться только свойства 1) и 4).

Рассмотрим множество всех проекций на ось $O x$ квадратов из $\mathscr{Q}^{\prime \prime}$, оставим среди этих проекций только максимальные по включению. Составим $\mathscr{Q}^{\prime}$ следуюшим образом. Для каждой проекции $\Delta_{k}^{n}=\left[2^{-k} n, 2^{-k}(n+1)\right]$, которую мы оставили, включим в покрытие $\mathscr{Q}^{\prime}$ все квадраты из одного вертикального ряда (при соответствующих $k$ и $n$ ), пересекающие множество $E$. При этом если $k=k_{0}$, считаем каждый из этих квадратов бельмм, а при $k<k_{0}-$ краснымм (напомним, что квадраты такого размера были красными и в покрытии $\left.\mathscr{Q}^{\prime \prime}\right)$.

Покажем, что покрытие $\mathscr{Q}^{\prime}$ - требуемое. Как уже отмечалось, оно обладает свойствами 2), 3) и 5). Докажем свойство 4). Как следует из построения, пря- 
мая, параллельная оси $O y$, может пересекать квадраты из $\mathscr{Q}^{\prime}$ только одного цвета и размера. Из свойства 3) следует, что число таких квадратов не превосходит абсолютной константы $A_{1}$, так как они не могут располагаться "слишком далеко" от $Г$. (Напомним, что в силу леммы $2.1,4)$ ломаная Г задается уравнением $y=\psi(x)$ с $\left|\psi^{\prime}(x)\right| \leqslant A$.) Выполнение условия $\sum^{\prime} d_{\text {кр }} \leqslant A\|\mu\|$ в 1 ) вытекает из (только что доказанного) свойства 4) и соответствующей оценки для проекций красных квадратов на ось $O x$ (которая, в свою очередь, следует из выполнения для покрытия $\mathscr{Q}^{\prime \prime}$ свойства, аналогичного 1)). Лемма доказана.

\section{§4. Доказательство ключевой оценки}

Сведем лемму 2.6 к доказательству оценки:

$$
\left|\int \Psi^{1}(z) d \mu_{1}(z)\right| \leqslant A\|\mu\|,
$$

где $\Psi^{1}(z)$ - функция из (4.8), представляющая собой сумму двух сингулярных интегралов (с ядрами $x / z^{2}$ и $y / z^{2}$ ) от ограниченных функций, заданных на липшицевой кривой, а $\mu_{1}$ - подходящая регуляризация меры $\mu$. Остаток, получающийся при переходе от $\mu \mathrm{k} \mu_{1}$ и от $\Psi$ к $\Psi^{1}$, оценим соответственно в леммах 4.1 и 4.2.

Для определения $\mu_{1}$ будет удобно видоизменить квадраты $D_{g}$ и $D_{b}$ из леммы 2.3. Пусть $D=Q_{k}^{n, l}$-произвольный квадрат леммы 2.3. Рассмотрим для него следующий (вообше говоря, незамкнутый) квадрат $\widetilde{D}$ с тем же множеством внутренних точек: $\widetilde{D}=\left[n 2^{-k},(n+1) 2^{-k}\right) \times\left[l 2^{-k},(l+1) 2^{-k}\right)$, если одновременно выполняются неравенства $(n+1) 2^{-k}<2 u(l+1) 2^{-k}<2$, а если какое-либо из этих неравенств не выполняется, то соответствующий полуинтервал (для $x$ или у) замыкается справа. Ясно, что все полученные квадраты в объединении составляют $D_{0}$, однако, в отличие от квадратов леммы 2.3 , они попарно не пересекаются.

Теперь определим меру $\mu_{1}$. Для любого квадрата $D$ из леммы 2.3 сужение $\mu_{1}$ на $D$ - мера, равномерно распределенная в квадрате $0.5 D$, причем

$$
\int_{\widetilde{D}} d \mu=\int_{0.5 D} d \mu_{1} .
$$

Ясно, что $\left\|\mu_{1}\right\| \leqslant\|\mu\|$.

Нетрудно показать, воспользовавшись условием 2) леммы 2.3, что для любого круга $B(z, r)$ выполнено условие:

$$
\left\|\mu_{1}\right\|(B(z, r)) \leqslant A r .
$$

ЛЕмма 4.1. Для функиии $\Psi(z)$ из (2.4) выполнена оценка:

$$
\left|\int \Psi(z) d\left(\mu-\mu_{1}\right)(z)\right| \leqslant A\|\mu\| .
$$

ДоКАЗАТЕЛЬСТво ЛЕммы 4.1. Для любого квадрата $Q$ из покрытия $\mathscr{Q}^{\prime}$ определим число $\omega_{Q}$. Пусть $\omega_{Q}=1$, если $Q$ красный, $u \omega_{Q}=\omega\left(2^{-k_{0}}\right)$, если $Q$ бельй. Тогда в силу леммы 1.1 для каждой функции $\Psi_{j}$ из $(2.4)$, соответствующей квадрату $Q_{j}$ из $\mathscr{Q}^{\prime}$, выполнено условие: $\left|\Psi_{j}(z)\right| \leqslant A \omega_{Q_{j}}$ для всех $z \in \mathbb{C}$. 
Прежде всего из условия 1) леммы 2.5 и (4.2) непосредственно следует:

$$
\left|\int_{3 Q_{j}} \Psi_{j}(z) d\left(\mu-\mu_{1}\right)(z)\right| \leqslant A \omega_{Q_{j}} d\left(Q_{j}\right)
$$

откуда в силу условия 2) леммы 2.5 получим:

$$
\sum_{j}\left|\int_{3 Q_{j}} \Psi_{j}(z) d\left(\mu-\mu_{1}\right)(z)\right| \leqslant A_{1}\|\mu\| .
$$

Таким образом, для доказательства (4.3) осталось установить оценку:

$$
\sum_{j}\left|\int_{D_{0} \backslash 3 Q_{j}} \Psi_{j}(z) d\left(\mu-\mu_{1}\right)(z)\right| \leqslant A_{1}\|\mu\| .
$$

Заметим, что если для квадрата $D$ из леммы 2.3 выполнено условие

$$
\operatorname{dist}\left(Q_{j}, D\right)<d\left(Q_{j}\right)
$$

то в силу леммы $2.4,2)$ обязательно $D \subset 3 Q_{j}$. Поэтому для каждого квадрата $Q_{j}$ рассмотрим только такие квадраты $D$ из леммы 2.3, что $\operatorname{dist}\left(Q_{j}, D\right) \geqslant d\left(Q_{j}\right)$. Пусть $t_{j}$ и $z_{D}$ - центры соответственно $Q_{j}$ и $D, z$ - произвольная точка $D$. Из леммы 1.1 (напомним, что $c_{0}\left(\Psi_{j}\right)=0$ ), условия 2) леммы 2.4 (в силу которого с учетом $\operatorname{dist}\left(Q_{j}, D\right) \geqslant d\left(Q_{j}\right)$ расстояния $\operatorname{dist}\left(Q_{j}, D\right)$ и $\left|t_{j}-z_{D}\right|$ "примерно равны") и теоремы Лагранжа получим:

$$
\left|\Psi_{j}(z)-\Psi_{j}\left(z_{D}\right)\right| \leqslant A \omega_{Q_{j}} \frac{d\left(Q_{j}\right) d(D)}{\left|t_{j}-z_{D}\right|^{2}}
$$

Так как $\int_{\widetilde{D}} d\left(\mu-\mu_{1}\right)(z)=0$, отсюда вытекает следующая оценка:

$$
\left|\int_{\widetilde{D}} \Psi_{j}(z) d\left(\mu-\mu_{1}\right)(z)\right| \leqslant A \omega_{Q_{j}}\|\mu\|(D) \frac{d\left(Q_{j}\right) d(D)}{\left|t_{j}-z_{D}\right|^{2}} .
$$

Зафиксируем квадрат $D$ из леммы 2.3 и просуммируем (4.5) по множеству $J(D)$ таких номеров $j$, что $\operatorname{dist}\left(Q_{j}, D\right) \geqslant d\left(Q_{j}\right)$. Так как в силу условия 4$)$ леммы 2.4 число квадратов $Q_{j}$ из одного вертикального ряда ограничено сверху абсолютной константой, то:

$$
\sum_{j \in J(D)}\left|\int_{\widetilde{D}} \Psi_{j}(z) d\left(\mu-\mu_{1}\right)(z)\right| \leqslant A\|\mu\|(D) d(D) \int_{d(D)}^{+\infty} \frac{d x}{x^{2}}=A\|\mu\|(D) .
$$

Отсюда, суммируя по всем квадратам $D$ леммы 2.3 , получим оценку:

$$
\sum_{\{D\}} \sum_{j \in J(D)}\left|\int_{\widetilde{D}} \Psi_{j}(z) d\left(\mu-\mu_{1}\right)(z)\right| \leqslant A_{1}\|\mu\|
$$

из которой изменением порядка суммирования получается (4.4), а значит, и (4.3). Лемма 4.1 доказана. 
Прежде чем определить функцию $\Psi^{1}$, будет удобно несколько “упростить" ломаную $\Gamma$. Рассмотрим произвольный квадрат $Q$ покрытия $\mathscr{Q}^{\prime}$. Если внутри $Q$ содержится более одной вершины $\Gamma$, то оставим произвольно из этих вершин только одну. Проведем ломаную через все оставшиеся вершины (последовательно, по возрастанию $x$ ), а за крайние вершины продолжим ее условием $y=$ const. Полученную ломаную обозначим $\Gamma_{1}$ и пусть $y=\psi_{1}(x)$ - ее уравнение. Ясно (в силу свойств $Г$ и покрытия $\mathscr{Q}^{\prime}$ из леммы 2.4 ), что для ломаной $\Gamma_{1}$ выполнено условие $\left|\psi_{1}^{\prime}(x)\right| \leqslant A$, а для любого квадрата $Q$ из $\mathscr{Q}^{\prime} \operatorname{dist}\left(\Gamma_{1}, Q\right) \leqslant A d(Q)$. При этом проекция $Q$ на $\Gamma_{1}$ в направлении оси $O y$ содержит прямолинейньй отрезок длины, не меньшей $0.5 d(Q)$.

Теперь вернемся к построению функции $\Psi^{1}(z)$. Для каждой функции $\Psi_{j}(z)$ из (2.4) в формуле (4.6) определим видоизмененную функцию $\Psi_{j}^{1}(z)$. Пусть $L_{j}-$ проекция квадрата $Q_{j}$ на ломаную $\Gamma_{1}$ в направлении оси $O y, d s-$ элемент длины $\Gamma_{1}$.

Рассмотрим функции $v_{x}^{j}(s)$ и $v_{y}^{j}(s)$, равнье нулю вне $L_{j}$ и обладающие следуюшими свойствами 1)-3):

1) $\max \left(\left|v_{x}^{j}(s)\right|,\left|v_{y}^{j}(s)\right|\right) \leqslant A \omega_{Q_{j}}$ для всех $s$;

2) $\int v_{x}^{j}(s) d s=c_{x}\left(\Psi_{j}\right), \quad \int v_{y}^{j}(s) d s=c_{y}\left(\Psi_{j}\right)$;

3)

$\max \left(\sup _{z \in \mathbb{C}} \mid\right.$ V.P. $\left.\int_{L_{j}} \frac{x-\tau(s)}{(z-t(s))^{2}} v_{x}^{j}(s) d s\left|, \sup _{z \in \mathbb{C}}\right| \operatorname{V} \cdot P \cdot \int_{L_{j}} \frac{y-\nu(s)}{(z-t(s))^{2}} v_{y}^{j}(s) d s \mid\right) \leqslant A \omega_{Q_{j}}$,

где $t(s)$ - соответствующая s точка на $\Gamma_{1}, \tau(s)=\operatorname{Re} t(s), \nu(s)=\operatorname{Im} t(s)$. При $z=x+i y \in \Gamma_{1}$ сингулярные интегралы здесь и ниже (для определенности) понимаются в смыссле главного значения.

Сушествование таких функций $v_{x}^{j}(s)$ и $v_{y}^{j}(s)$ непосредственно вытекает из $(2.5)$ и из того факта, что $L_{j}$ содержит некоторьй прямолинейньй отрезок $l_{j}$ длины, не меньшей $0.5 d\left(Q_{j}\right)$ ( $v_{x}^{j}$ и $v_{y}^{j}$-характеристические функции отрезка $0.5 l_{j}$, сглаженные вблизи его концов и подходяшим образом нормированные).

Рассмотрим следуюшие функции:

$$
\Psi_{j}^{1}(z)=\mathrm{V} . \mathrm{P} . \int_{L_{j}} \frac{x-\tau(s)}{(z-t(s))^{2}} v_{x}^{j}(s) d s+\mathrm{V} . \mathrm{P} . \int_{L_{j}} \frac{y-\nu(s)}{(z-t(s))^{2}} v_{y}^{j}(s) d s .
$$

Ясно, что функции $\Psi_{j}^{1}$ бианалитичны вне $L_{j}$ и $c_{0}\left(\Psi_{j}^{1}\right)=0$. Из свойства 2) функций $v_{x}^{j}(s)$ и $v_{y}^{j}(s)$ следует, что $c_{x}\left(\Psi_{j}^{1}\right)=c_{x}\left(\Psi_{j}\right)$ и $c_{y}\left(\Psi_{j}^{1}\right)=c_{y}\left(\Psi_{j}\right)$. Из $\operatorname{dist}\left(Q_{j}, \Gamma_{1}\right) \leqslant A d\left(Q_{j}\right)$ и леммы $\left.1.1,4\right)$ (где $k=2$ ) вытекает, что для каждого $j$ :

$$
\left|\Psi_{j}(z)-\Psi_{j}^{1}(z)\right| \leqslant A r_{j}(z),
$$

где $r_{j}(z)$ - следующие функции:

$$
r_{j}(z)=\omega_{Q_{j}} \min \left(1, \frac{\left(d\left(Q_{j}\right)\right)^{2}}{\left|z-t_{j}\right|^{2}}\right) .
$$


ЛЕмма 4.2. Выполняется оценка:

$$
\sum_{j} \int r_{j}(z)\left|d \mu_{1}(z)\right| \leqslant A\|\mu\|
$$

ДоКАЗАТЕЛЬСТво ЛЕмМы 4.2. В силу условия 2) леммы 2.5 достаточно для каждого $j$ установить оценку:

$$
\int r_{j}(z)\left|d \mu_{1}(z)\right| \leqslant A_{1} \omega_{Q_{j}} d\left(Q_{j}\right)
$$

Но из (4.2) и (4.7), разбивая интеграл на сумму интегралов по $B\left(t_{j}, d\left(Q_{j}\right)\right)$ и кольцам $B\left(t_{j}, 2^{k+1} d\left(Q_{j}\right)\right) \backslash B\left(t_{j}, 2^{k} d\left(Q_{j}\right)\right), k=0,1, \ldots$, получим:

$$
\begin{aligned}
\int r_{j}(z)\left|d \mu_{1}(z)\right| & \leqslant A_{2} \omega_{Q_{j}} \sum_{k=1}^{\infty} \frac{\left\|\mu_{1}\right\|\left(B\left(t_{j}, 2^{k} d\left(Q_{j}\right)\right)\right)}{2^{2 k}} \\
& \leqslant A_{1} \omega_{Q_{j}} d\left(Q_{j}\right) \sum_{k=1}^{\infty} \frac{1}{2^{k}}=A_{1} \omega_{Q_{j}} d\left(Q_{j}\right) .
\end{aligned}
$$

Лемма 4.2 доказана.

Теперь рассмотрим следующую функцию:

$$
\Psi^{1}(z)=\sum_{j} \Psi_{j}^{1}(z)
$$

В силу (4.6)

$$
\begin{aligned}
\Psi^{1}(z)= & \text { V.P. } \int_{\Gamma_{1}} \frac{x-\tau(s)}{(z-t(s))^{2}}\left(v_{1 x}(s)+v_{2 x}(s)\right) d s \\
& + \text { V.P. } \int_{\Gamma_{1}} \frac{y-\nu(s)}{(z-t(s))^{2}}\left(v_{1 y}(s)+v_{2 y}(s)\right) d s,
\end{aligned}
$$

где $v_{1 x} u v_{1 y}$ - суммы по $j$ соответственно всех $v_{x}^{j}(s) u v_{y}^{j}(s)$, полученных при проектировании на $\Gamma_{1}$ красных квадратов; $v_{2 x}$ и $v_{2 y}$ - такие же функиии, но для бельх квадратов.

В силу леммы 4.2

$$
\left|\int\left(\Psi(z)-\Psi^{1}(z)\right) d \mu_{1}(z)\right| \leqslant A\|\mu\|
$$

и поэтому для доказательства леммы 2.6 достаточно установить оценку (4.1).

Применив неравенство Коши-Буняковского, получим:

$$
\left|\int \Psi^{1}(z) d \mu_{1}(z)\right| \leqslant \sqrt{\left\|\mu_{1}\right\|} \sqrt{\int\left|\Psi^{1}(z)\right|^{2} d\left|\mu_{1}(z)\right|} .
$$

Так как $\left\|\mu_{1}\right\| \leqslant\|\mu\|$, оценка (4.1) сводится к следующей:

$$
\int\left|\Psi^{1}(z)\right|^{2} d\left|\mu_{1}(z)\right| \leqslant A_{1}\|\mu\|
$$


Каждый из двух интегралов, составляющих $\Psi^{1}(z)$, представляет собой сингулярньй интеграл с антисимметричным ядром Кальдерона-Зигмунда, аналитическим в $\mathbb{C} \backslash\{0\}\left(x / z^{2}\right.$ и $y / z^{2}$ соответственно). Поэтому (например, [17; ч. 3 , п. 4 , теорема 4.2$]$ ) операторы, соответствуюшие этим интегралам, ограничены в пространстве $L_{2}(d s)$ на $\Gamma_{1}$, т.е.

$$
\int_{\Gamma_{1}}\left|\Psi^{1}(z(s))\right|^{2} d s \leqslant A \int_{\Gamma_{1}}\left(\left|v_{1 x}(s)+v_{2 x}(s)\right|^{2}+\left|v_{1 y}(s)+v_{2 y}(s)\right|^{2}\right) d s .
$$

(Напомним, что $\Gamma_{1}-$ липшицева кривая, заданная уравнением $y=\psi_{1}(x)$, причем $\left|\psi_{1}^{\prime}(x)\right| \leqslant A$.)

Отсюда и из (4.2) вытекает, например, в силу леммы 2.5 из [17; ч. 3, п. 2], что

$$
\int\left|\Psi^{1}(z)\right|^{2} d\left|\mu_{1}(z)\right| \leqslant A \int_{\Gamma_{1}}\left(\left|v_{1 x}(s)+v_{2 x}(s)\right|^{2}+\left|v_{1 y}(s)+v_{2 y}(s)\right|^{2}\right) d s .
$$

(В лемме 2.5 из [17; ч. 3, п. 2] речь, в частности, идет об ограниченности максимального сингулярного интегрального оператора, действующего из $L_{2}(d s)$ в $L_{2}\left(d \mu_{1}\right)$ при условии $\left\|\mu_{1}\right\|(B(z, r)) \leqslant A r$ для любого круга $B(z, r)$.)

Таким образом, для доказательства (4.9) осталось показать, что

$$
\int_{\Gamma_{1}}\left(\left|v_{1 x}(s)+v_{2 x}(s)\right|^{2}+\left|v_{1 y}(s)+v_{2 y}(s)\right|^{2}\right) d s \leqslant A_{2}\|\mu\| .
$$

Напомним, что в силу условия 4) леммы 2.4 кратность попарных пересечений носителей $v_{x}^{j}$ и $v_{y}^{j}$ при различных $j$ не превосходит абсолютной константы. Поэтому из свойства 1) функций $v_{x}^{j}$ и $v_{y}^{j}$ получим: $\max \left(\left|v_{1 x}\right|,\left|v_{1 y}\right|\right) \leqslant A$ и $\max \left(\left|v_{2 x}\right|,\left|v_{2 y}\right|\right) \leqslant$ $A \omega\left(2^{-k_{0}}\right)$. Отсюда вытекает:

$$
\begin{aligned}
& \int_{\Gamma_{1}}\left(\left|v_{1 x}(s)\right|^{2}+\left|v_{1 y}(s)\right|^{2}\right) d s \leqslant A_{3} \sum^{\prime} d_{\text {кр }}, \\
& \int_{\Gamma_{1}}\left(\left|v_{2 x}(s)\right|^{2}+\left|v_{2 y}(s)\right|^{2}\right) d s \leqslant A_{3} \omega\left(2^{-k_{0}}\right) \sum^{\prime} d_{\text {бел. }}
\end{aligned}
$$

Применив лемму 2.5, 2), получим (4.10) и, следовательно, нужную оценку (4.1). Лемма 2.6 доказана.

\section{Список литературы}

1. Балк М.Б. Полианалитические функции и их обобщения // Итоги науки и техники. Фундамент. напр. Т. 85. М.: ВИНИТИ, 1991. С. 187-246.

2. Verdera $J$. On the uniform approximation problem for the square of the Cauchy-Riemann operator // Pacific J. Math. 1993. V. 159. P. 379-396.

3. Verdera J. The uniform approximation problem for the square of the Cauchy-Riemann operator // Lecture Notes in Math. 1994. V. 1574. P. 122-123.

4. Витушкин А. Г. Аналитическая емкость множеств в задачах теории приближений // УМH. 1967. Т. 22. №6. С. 141-199.

5. Келдыш М. В. О разрешимости и устойчивости задачи Дирихле // УМН. 1941. №8. C. $171-231$.

6. Deny J. Systémes totaux de functions harmoniques // Ann. Inst. Fourier (Grenoble). 1949. V. 1. P. 103-113. 
7. Trent T., Wang J. L. Uniform approximation by rational modules on nowhere dense sets // Proc. Amer. Math. Soc. 1981. V. 81. P. 62-64.

8. Carmona J. J. Mergelyan approximation theorem for rational modules // J. Approx. Theory. 1985. V. 44. P. 113-126.

9. Wang J. L. A localization operator for rational modules // Rocky Mountain J. Math. 1989. V. 19. № 4. P. 999-1002.

10. Мазалов $M$. A. Равномерноеприближение функций, непрерьвных на произвольном компакте в $\mathbb{C}$ и аналитических внутри компакта, функциями, бианалитическими в его окрестности // Матем. заметки. 2001. Т. 69. № 2. С. 245-261.

11. Verdera J. $C^{m}$ approximation by solutions of elliptic equations, and Calderon-Zygmund operators // Duke Math. J. 1987. V. 55. P. 157-187.

12. Парамонов П. В. О гармонических приближениях в $\mathbb{C}^{1}$-норме // Матем. сб. 1990. T. 181. № 10. С. 1341-1365.

13. Стейн И. Сингулярные интегралы и диффференциальные свойства функиций. М.: Мир, 1973.

14. Парамонов П. В. Некоторые новые критерии равномерной приближаемости функций рациональньми дробями // Матем. сб. 1995. Т. 186. №9. С. 97-112.

15. Nguyen Xuan Uy. An extremal problem on singular integrals // Amer. J. Math. 1980. V. 102. № 2. P. 279-290.

16. Harvey R., Polking J. Removable singularities of solutions of linear partial differential equations // Acta Math. 1970. V. 125. P. 39-56.

17. David G. Wavelets and singular integrals on curves and surfaces. New York: Springer-Verlag, 1991. (Lecture Notes in Math. V. 1465.)

Военншй университет Войсковой ПВО

Поступила в редакцию

Вооруженных сил РФ

20.10 .2003

E-mail: mazalov@sci.smolensk.ru 